\title{
PERICULUM REI VENDITAE E PERICULUM DOTIS AESTIMATAE*
}

\author{
Eduardo César Silveira Vita Marchi \\ Professor Titular do Departamento de Direito Civil da \\ Faculdade de Direito da Universidade de São Paulo
}

Resumo:

A ampla maioria da doutrina romanística, como se sabe, atribui ao Direito Romano clássico o princípio periculum est emptoris, pelo qual o risco ou o periculum (decorrente do perecimento casual da coisa vendida) caberia, já desde a celebração do contrato (e, portanto, antes mesmo da entrega da mercadoria), ao comprador.

O autor, neste artigo, contrariando a communis opinio, procura, através da análise de fragmentos em matéria de dos aestimata, demonstrar que, ao contrário, no Direito Romano clássico, a chamada "solução germânica" (adotada também pelo Código Civil brasileiro), na qual o comprador suporta o risco apenas a partir do momento da traditio ou entrega da merx, teria exercido um papel fundamental em não poucos textos.

\begin{abstract}
:
The great majority of the romanistic literature ascribes to classical Roman Law the periculum est emptoris doctrine, according to which the risk or periculum (i.e., the chance or possibility that the object of the sale might accidentally perish) had to be immediately born by the purchaser once the sale was perfect (and, accordingly, even before the goods had been actually conveyed to him)

Opposing the communis opinio, the author of the present essay tries to demonstrate, by the analysis of several passages of the Roman sources concerning the dos aestimata, that, on the contrary, in classical law the so-called "german solution" (also adopted in the brazilian Civil Code) ---according to which the purchaser only had to bear the risk after the traditio or delivery of the merx--- had a fundamental significance in not few texts of the Roman jurisprudence.
\end{abstract}

Unitermos: risco de compra; comprador; entrega de mercadoria.

* Ringrazio la prestigiosa 'Alexander von Humboldt-Stiftung' per la borsa di ricerca che mi ha consentito di scrivere questo articolo. Ringrazio anche il 'Leopold Wenger-Institut' della Facoltà di Giurisprudenza dell'Università di Monaco, in cui, nel corso dell'anno 1993, questa ricerca è stata condotta, ed in particolare ringrazio il Prof. Dieter Nörr alla cui disponibilità devo molto, ed i suoi assistenti Tiziana Chiusi, Wolfgang Kaiser e Hans-Dieter Spengler. 
Sommario:

I Introduzione

II - Il problema del Periculum Rei Venditae

II. 1 - Linee generali

II.1.1 Proposta di cambiamento di impostazione

III - Aspetti generali del problema del Periculum Dotis Aestimatae

III. 1 La dos Aestimata: Nozione

III.2 - Il Periculum Dotis Aestimatae: Premesse

IV L'esegesi dei passi "Clavis Huius Materiae"

IV.1 - L'esegesi di Ulp. 34 ad ed., D. 23,3,14

IV.1.1 Difetti formali

IV.1.2 - L'argumentum e contrario

IV.1.3 - La mora della moglie: significato ed effetto

IV.2 - L'esegesi di Pomponio 14 ad. Sab., D. 23,3,15

IV.2.1 Difetti formali e sostanziali

IV.2.2 - Il contesto originale della fattispecie e l'ipotesi di Haymann di "Annahmeverzug" del marito-compratore

V Conclusione

I - Introduzione

Dopo la conclusione della compravendita, ed il suo "perfezionamento", 1 ma prima della consegna della cosa venduta al compratore,

I. In tema di emptio venditio la dottrina è solita (cf., ad esempio, Wieacker, Die juristische Sekunde. Zur Legitimation der Konstruktionsjurisprudenz, in Festschrift für E. Wolf 60. Geburtstag, 1962, p. 432, Kaser, Das römische Privatrecht I, 1971, pp. 552-553, Knütel, Kauf und Pacht bei Abzahlungsgeschäften im römischen Recht, in Festschrift Kaser z. 65. Geburtstag, 1973, p. 44, Peters, Rücktrittsvorbehalte des römischen Kaufrechts, 1973, p. 147, e Guarino, Diritto Privato Romano, 1984, pp. 788-799) distinguere tra il momento di "conclusione" (o "celebrazione") del contratto e quello del suo "perfezionamento": quest'ultimo, che sarebbe posteriore a quello della "conclusione", corrisponderebbe, come risulta da Paul. 33 ad ed., D. 18,6,8 pr. ("...et si id quod venierit appareat quid quale quantum sit, sit et pretium, et pure venit, perfecta est emptio..."), a casi eccezionali --come quelli della compravendita sottoposta a condizione sospensiva (D. 18,6,8 pr.), o in cui l'oggetto non è chiaramente individuato, come la compravendita "aus beschränktem Vorrat" (Frag.Vat. 16 und C. 4,48,2,1-2), oppure "mit Zumessungserfordernis" od ancora "mit Degustationsabrede" (Gai. 10 ad ed. prov., D. 18,1,35,5-7)--, nei quali si manifesta l'esigenza di fissare il quid quale quantum affinchè il negozio possa produre tutti $\mathrm{i}$ suoi effetti (o più specificamente, affinchè --secondo la communis opinio, come si vedrà di seguito-- in questi casi eccezionali il principio periculum est emptoris abbia applicazione: "perfecta emptione periculum ad emptorem respicit" - D. 18,6,8 pr.). 
quest'ultima viene a perire o deteriorarsi per caso fortuito o forza maggiore e ciò̀ senza colpa delle parti. Chi dovrà farsi carico del danno? Il venditore? Il compratore? In altre parole: deve quest'ultimo pagare il prezzo, sia nel caso che egli non possa più ricevere la (ormai inesistente) merx, che in quello in cui la riceve, ma deteriorata?

A questa questione, che riguarda appunto il problema del periculum o rischio $^{2}$ nella compravendita, la compilazione giustinianea sembra rispondere richiamando il (a prima vista generalizzato) principio periculum est emptoris: il compratore deve sopportare il rischio del casuale perimento o deterioramento della cosa venduta ${ }^{3}$ già a partire dalla conclusione del contratto.

L'ampia maggioranza della dottrina --come è notorio-- suppone che questa sia anche stata la soluzione accolta dal diritto classico. Il principio periculum est emptoris sarebbe dunque genuino.

Tra i passi richiamati da questa communis opinio come testimonianze -secondo le parole di Arangio-Ruiz ${ }^{4}$-- "così esplicite e fededegne" da non consentire dubbi sulla classicità del principio per cui "il rischio della cosa passa al compratore al momento del contratto, anche se non vi sia stata tradizione" (periculum est emptoris), sono elencati due frammenti, posti l'uno dopo l'altro nella Compilazione, la cui importanza è stata sempre (a nostro avviso) un po' trascurata dalla dottrina: Ulp. 34 ad ed., D. 23,3,14, e Pomp. 14 ad Sab., D. 23,3,15.

Essi riguardono il problema del periculum nella cosiddetta dos aestimata, una figura speciale di costituzione di dote, che --come si vedrà nella seconda parte di questo lavoro-- viene equiparata nella Compilazione alla compravendita, da cui, in tesi, deriverebbe l'esigenza di applicare le stesse regole.

2. Il vocabulo periculum, anche se impiegato in questa frase come sinonino di rischio in generale, non presenta nelle fonti romane -al contrario che nella dogmatica moderna- un significato univoco; l'accertamento del senso del termine periculum nelle nostre fonti costituisce appunto uno dei punti cruciali e di maggior difficoltà nelle ricerche in tema di periculum rei venditae; su esso, cf. in speciale Miquel, "Periculum locatoris", in ZSS 81 (1964), pp. 134 ss.

3. Al contrario della dottrina italiana, che suole impiegare l'espressione periculum rei venditae o "rischio derivato del perimento o deterioramento della cosa venduta" tanto quando ci si riferisce alla posizione del venditore quanto del compratore, la dottrina tedesca invece, per quanto riguarda il rischio sopportato da quest'ultimo e cioè l'emptor, preferisce adoperare l'espressione "Preisgefahr" o "rischio del prezzo"

4. La compravendita in diritto romano II, Napoli, 1956, p. 266. 
II Il problema del Periculum Rei Venditae

II.1 - Linee generali

La communis opinio tradizionale ha da sempre --come si è già detto d'inizio-- riconosciuto la classicità del principio periculum est emptoris, nonostante la presenza di significative voci in senso diametralmente opposto - periculum est venditoris - quali, accanto a quella di Arnò, ${ }^{5}$ le prese di posizione del calibro di Haymann $^{6}$ e Betti, ${ }^{7}$ che attribuiscono la prima soluzione al diritto giustinianeo. Non sono mancati pure tentativi di mitigazione del principio, tra cui il più riuscito è quello di Seckel-Levy, ${ }^{8}$ che, senza rifiutarne la classicità, limitano la sua portata ai casi di vis maior (tramite il ricorso alla "Custodialehre"), nonchè al raggiungere dello stadio dell'emptio perfecta ${ }^{9}$ dal negozio, tesi accolta dalla communis opinio attuale.

Il riconoscimento della classicità del principio ha comunque sempre prevalso in dottrina; anzi, se si guarda agli studi più recenti sul nostro tema, quali quelli di Ernst ${ }^{10}$ e Thielmann, ${ }^{1 "}$ ci si accorge addirittura di una tendenza alla radicalizzazione di questa tesi, a supporsi che in epoca classica non era consentita

5. La teorica del periculum rei venditae nel diritto romano classico in Giurisprudenza Italiana 49 (1897), pp. 209 ss.; Note minime sul § 3 Inst. 3,23 in $A G 62$ (1899), pp. 540 ss.; Commento ad Cod. 4, 48, 1 in $A G 65$ (1900), pp. 301 ss.; La const. 2 C. 4, 48 nella Summa Perusina in AG 68 (1902), pp. 429 ss.; La massima 'dolus auctoris bona fide emptori non nocet' nella c. 3 C. 4,48 in Studi Scialoja I, 1905, pp. 341 ss.; Di una interpolazione certa nella c. 4 C. 4,48 in Mélanges Fitting, 1907, pp. 29 ss.; La costituzione ultima del Codice de periculo et commodo rei venditae in Studi Brugi, 1910, p. 154; e Sul c. 23 dei Fragmenta Vaticana in Memorie della R.Academia di Scienze Lettere ed Arti in Modena 9 (1910), pp. 73 ss.

6. Textkritische Studien zum römischen Obligationrecht. I- Über Haftung für custodia in ZSS 40 (1919), pp. 167-350, II - "Periculum est emptoris" in ZSS 41 (1920), pp. 44-185, e Zur klassizität des "periculum emptoris" in ZSS 48 (1928), pp. 314-418. L'autore fu seguito da Konstantinovitch, Le "periculum rei venditae" en droit romain, Lyon, 1923.

7. "Periculum" Problema del rischio contrattuale in diritto romano classico e giustinianeo, in Studi De Francisci I, Milano, 1956, pp. 131-197; Istituzioni di diritto romano II, I, Padova, 1960, pp. 410-421; Zum Problem der Gefahrtragung bei zweiseitig verpflichtenden Verträgen, in ZSS 82 (1965), pp. 1-23. L'autore fu seguito da Sargenti, Rischio contrattuale (diritto romano), in ED 40 (1989), pp. 1.126-1.132.

8. Die Gefahrtragung beim Kauf im klassichen römischen Recht, in ZSS 47 (1927), pp. 117-263.

9. Cf. nota I sopra.

10. "Periculum est emptoris" in ZSS 99 (1982), pp. 216-248.

11. Traditio und Gefahrübergang in ZSS 106 (1989), pp. 292-326. 
assolutamente alcuna eccezione o deroga alla regola secondo la quale il pericolo del fortuito perimento della cosa oggetto della compravendita, era attribuito al compratore già a partire dalla conclusione del contrato. Infatti, il primo autore contesta addirittura che le decisioni riguardanti la vendita sottoposta a condizione (Frag.Vat.23 = C.4,48,5 und Paul. 33 ad ed., D. 18,6,8 pr.), la vendita "aus beschränktem Vorrat" (Frag.Vat. 16 und C. 4,48,2,1-2), la vendita "mit Zumessungserfordernis" (Gai. 10 ad. ed. prov., D. 18,1,35,5-7), e quella (specialmente di vino) "mit Degustationsabrede" (Ulp. 28 Sab., D. 18,6,1 pr. und D. $18,6,4$ pr. und 1) abbiano contenuto una qualsiasi eccezione alla regola del periculum emptoris. Egli infatti fa coincidere la emptio perfecta con il venire in essere o l'esistenza del contratto di compravendita. ${ }^{12}$ Nella stessa direzione Thielmann nella sua indagine sui rapporti tra traditio e passaggio del rischio, si sforza, con scarso sucesso, di provare che Afric. 8 quaest., D. 19,2,33 e Paul. 3 epit. Alf., D. 18,6,13 und 15 pr.-1 (in relazione ai famosi casi del fundus publicatus, dei lecti empti e della materia empta) non sono in contraddizione con la regola periculum est emptoris: secondo l'autore D. 19,2,33 non avrebbe potuto essere inserito come eccezione a quella regola, perchè la fattispecie lascia riconoscere pochissime particolarità. Dal canto suo il gruppo di passi D. 18,6,13 und 15 pr.-1 riguarderebbe la responsabilità del compratore per culpa e per lesione dell'obbligo di custodia e per questo non avrebbe niente a che fare con il principio periculum est emptoris. ${ }^{13}$

Oltre questa tendenza, si può anche notare (e criticare) nella dottrina più recente, come esattamente sottolinea Talamanca, ${ }^{14}$ la prevalenza di una "soluzione monistica" della ripartizione del rischio contrattuale nella compravendita,

12. Senza volere -come si è detto- rifiutare la classicità del principio periculum est emptoris (e in ciò essendo d'accordo con la communis opinio) Ernst ha però proposto un'altra spiegazione del concetto di emptio perfecta. La "perfezione" indicherebbe, secondo l'autore, "den Zeitpunkt, zu dem der Kauf als gültiger Rechtsakt überhaupt erst zustande kommt" ("Periculum" cit. [nota 10 sopra], p. 237). Prima della fissazione del quid quale quantum (il che significa prima della "perfezione") la vendita sarebbe ritenuta un negozio giuridico ancora inesistente e perciò sarebe un ato giuridico assolutamente non prodottivo di effetti. Da questo consegue che la "perfezione" della vendita non sarebbe un pressuposto dell'applicazione del principio periculum est emptoris, bensi del venire in esistenza dello stesso contratto di vendita come negozio giuridico efficace e valido. Conseguentemente i Romani -conclude Ernst- non avrebbero ammesso alcuna eccezione o limitazione della regola periculum est emptoris.

13. A tal proposito si deve osservare che Thielmann riprende parzialmente in questo punto una soluzione già proposta nel secolo scorso dalla "Pandektenharmonistik"

14. Vendita (diritto romano), in Enciclopedia del Diritto 46 (1993), p. 451. 
e cioè l'insistenza nel costruire una "disciplina uniforme" basata sul principio periculum est emptoris e sullo schema concettuale della emptio perfecta.

\section{II.1.1. Proposta di cambiamento di impostazione}

Anche se, ad un primo sguardo, sono molti i passi in cui si applica il (preteso) principio periculum est emptoris, ci pare, dopo un riesame in generale dei passi riguardanti il problema del periculum rei venditae, seguito dallo studio specifico (sopra fatto) di quelli principali in materia di periculum dotis aestimatae, che la chiave di lettura debba essere cambiata. La ampia maggioranza della dottrina è solita dare per scontato il riconoscimento della regola periculum est emptoris come un principio generale in tema di compravendita. Secondo noi invece questa regola raggiungerà la qualifica o forza di "principio generale" soltanto con Giustiniano. Cosa che non dovrebbe sorprendere, data la chiara presa di posizione dell'imperatore in Inst. $3,23,3,{ }^{15}$ passo che non ha --il che è molto significativo, come sempre osservato dalla dottrina contraria alla communis opinio-- corrispondenza nel manuale di Gaio.

É stato dunque (probabilmente) il diritto giustinianeo ad elevare tale regola alla posizione di "principio generale". In questo senso dunque, e cioè per quanto riguarda la ricostruzione della regola giustinianea di ripartizione del rischio contrattuale nella emptio venditio, la dottrina (ampiamente minoritaria) capitanata da Haymann e Betti ci sembra la più accettabile.

Però in quale misura od estensione siano arrivati i giustinianei alla fine di raggiungere questo oggettivo --e cioè entro quali limiti alcuni (pochi) testi --come

15. "Cum autem emptio et venditio contracta sint quod effici diximus, simulatque de pretio convenerit, 'cum sine scriptura res agitur', periculum rei venditae statim ad emptorem pertinet, tametsi adhuc ea res emptori tradita non sit. Itaque si homo mortuus sit vel aliqua parte corporis laesus fuerit, aut aedes totae aut aliqua ex parte incendio consumptae fuerint, aut fundus vi fluminis totus vel aliqua ex parte ablatus sit, sive etiam inundatione aquae aut arboribus turbine deiectis longe minor aut deterior esse coeperit: emptoris damnum est, cui necesse est, licet rem non fuerit nactus, pretium solvere. Quidquid enim sine dolo et culpa venditoris accidit, in eo venditor securus est. Sed et si post emptionem fundo aliquid per alluvionem accessit, ad emptoris commodum pertinet: nam et commodum eius esse debet. cuitus periculum est. 3 a. Si fugerit homo qui veniit aut subreptus fuerit, ita ut neque dolus neque culpa venditoris interveniat, animadvertendum erit, an custodiam eius usque ad traditionem venditor susceperit. Sane enim, si susceperit, ad ipsius periculum is casus pertinet: si non susceperit, securus erit. Idem et in ceteris animalibus ceterisque rebus intellegimus. Utique tamen vindicationem rei et condictionem exhibere debebit emptori, quia sane, qui rem nondum emptori tradidit, adhuc ipse dominus est. Idem est etiam de furti et de damni iniuriae actione. 
ad esempio Ulp. 32 ad ed., D.19,5,20,1-- siano da considerarsi rimaneggiati o da sottoporsi ad una duplex interpretatio--, resta comunque a nostro parere un problema aperto. Ad una prima analisi, questi testi forse rimageggiati sono apparsi di numero ridotto e di poca importanza: la quasi totalità dei passi (citati a sostegno della tesi dominante) invece, può essere a nostro avviso interpretata senza ricorso alla già citata "soluzione monistica" (che si basa sull'applicazione del preteso "principio generale" periculum est emptoris). Essi possono essere raggruppati --a seconda della fattispecie e del problema centrale che trattono-- nei seguenti gruppi:

a) i passi (la ampia maggioranza) in cui, in relazione alle determinate caratteristiche del caso, la "soluzione" --e non dunque il "principio generale"-periculum est emptoris viene impiegata (ad es., Paul. 33 ad ed., D. 18,6,8 pr.[= il testo più discusso per il nostro tema], Frag.Vat. 16, C. 4,48,2,1-2, e Gai. 10 ad ed. prov., D. 18,1,35,5-7);

b) i passi che non hanno a che vedere --essenzialmente-- con la questione del periculum rei venditae (ad es., Paul. 5 ad Sab., D. 18,6,7 pr., e Ulp. 7 dig. Iul. Scaev., D. 18,6,11);

c) i passi possibile oggetto di una duplex interpretatio (ad es., Pomp. 14 ad Sab., D. 23,3,15);

d) i passi da cui risulta indirettamente l'impiego della soluzione contraria periculum est venditoris, fondata verosimilmente sul principio della traditio (ad es., Iavol. 7 ex Cass., D. 18,6,17); e finalmente,

e) i passi da cui risulta direttamente l'impiego della soluzione opposta, basata sicuramente sul principio della traditio (ad es., Paul. 3 Alf. dig., D. 18,6,13 e 15 pr.-1, così come Afr. 8 quaest., D. 19,2,33).

Per quanto riguarda invece il diritto classico ci pare più esatto affrontare il problema impostandolo nel senso di cercare linee di soluzioni eventualmente differenziate a seconda dei diversi contesti problematici e delle differenti personalità dei giuristi, come giustamente ha proposto di recente il Talamanca, ${ }^{16}$ (riprendendo in parte comunque una tesi già in passato sostenuta da Rabel): ${ }^{17}$ "con ogni probabilità" afferma il maestro romano, "v'era un'articolazione

16. Cf. Vendita cit. (nota 14 sopra), p. 451 e 11.1538 ; cf. anche Istituzioni di diritto romano, Milano, 1990, p. 588.

17. Cf. Gefahrtragung beim Kauf, in ZSS 42 (1921), pp. 543-564 (specialmente p. 559): secondo Rabel i giuristi classici non avrebbero avuto una dottrina unitaria riguardo il problema del periculum 
di soluzioni, a seconda delle fattispecie e dei giuristi", tenendosi conto, ad es., "delle caratteristiche dell'oggetto e del potere di controllo su di esso" 18

In poche parole: in tema di periculum rei venditae quindi è probabile che l'ordinamento classico fosse contrassegnato da una "elastische Gebilde" Non ci sarebbe dunque in diritto classico un "principio generale" per cui il rischio era attribuito al compratore dal momento della conclusione del contratto. A seconda dunque dei singoli casi o fattispecie, si attribuiva il pericolo ora al compratore ora al venditore. ${ }^{19}$ Valgono qui le parole di Bürge, ${ }^{20}$ pur espresse riguardo un altro tema: "Bei der Durchsicht dieser zeitlich über einen grösseren Zeitraum gestreuten Quellen hat sich wieder einmal gezeigt, wie wenig sinnvoll es ist, juristische Quellentexte von vornherein in eine einheitliche historische Genese zwängen zu wollen. Die tatsächlichen Verhältnisse waren komplizierter und liessen manche Gestaltungsmöglichkeiten offen, die ohne weiteres nebeneinander Bestand haben konnten"

Molte delle dichiarazioni delle nostre fonti, come quelle (più frequenti) di attribuzione dei rischi al compratore --ad es., "quia evenit emptoris periculo est" -D. 23,3,15--, non dovrebbero dunque a priori indurre alla idea

rei venditae; il passaggio dei rischi dal venditore al compratore poteva cadere, a seconda dei casi, in momenti diversi, rappresentando la traditio soltanto il più tardo tra quelli; oltre a ciò, in un certo gruppo di casi, alcune specie di rischi --come quelli cagionati da terzi oppure causati da furto, contemplati in D.18,6,13-15-- non sarebbero stati inquadrati nello schema concettuale del periculum rei venditae, il quale pressupporrebbe appena i danni derivati da eventi naturali.

18. Questa valutazione differenziata, come precisamente ancora osserva il Talamanca, Vendita cit. (nota 14 sopra), p. 451, corrisponderebbe esattamente al metodo casistico dei giuristi romani ed al ius controversum.

19. L'accettazione di tali premesse sconsiglierebbe -in termini metodologici- l'impostazione delle richerche in tema di periculum rei venditue a livello di "trattazione generale", come hanno fatto molti autori fino adesso, specialmente in epoca meno recente (come ad. es. Haymann e Seckel-Levy): e cioè sarebbe preferibile che le ricerche sul nostro tema procedessero attraverso l'esame dei singoli passi a seconda delle fattispecie in essi trattate e del problema centrale discusso, al limite raggruppandoli quando possibile- a seconda del tipo de problema: ad es periculum dotis, compravendita condizionale, compravendita "aus beschränktem Vorrat", compravendita "mit Zumessungserfordernis" (oppure di modo ancora più ristretto, e.g. compravendita de vino) ecc.

D'altra parte una tale impostazione metodologica renderebbe possibile un controllo piú attento della letteratura. Una trattazione generale del problema del periculum, anche se limitato alla compravendita infatti --senza quindi il periculum in altri rapporti contrattuali, come soprattutto la locatio conductio, e fuori dei contratti di scambio, la societas-- si rivela sempre più difficile, dato l'enorme numero di lavori dedicati al tema, il che si compreende, dato il ruolo centrale rappresentato dal problema del periculum rei venditae in diritto privato romano.

20. Der Witz im antiken Seefrachtvertrag. Beobachtungen zur Vertragspraxis im antiken Mitlelmeerraum, in INDEX 22 (1994), p. 402. 
preconcetta di un "principio generale"

Tra questi diversi contesti problematici --che esigono, come si è detto, soluzioni differenziate-- sono specialmente da elencare non soltanto quelli in cui si manifesta la valenza casistica della bona fides, come sottolineato da Talamanca, ${ }^{21}$ oppure lo schema concettuale della emptio perfecta, come ammesso dalla communis opinio attuale, ma anche il ruolo fondamentale rappresentanto in alcuni passi dal principio cosiddetto della traditio --chiamato anche "germanico"--, ${ }^{22}$ in cui il compratore deve sopportare il rischio soltanto a partire dal momento della consegna della cosa venduta, soluzione riassunta nella regola periculum est venditoris.

In quest'ultimo senso, e cioè per quanto riguarda il ruolo cęntrale svolto dal principio della traditio nella soluzione di certi passi in materia di periculum rei venditae, s'impone qui ancora una volta la menzione dei discussi testi (già citati) di Paul. 3 Alf. dig., D. $18,6,13$ e 15 pr.-1 ${ }^{23}$ noncchè Afr. 8 quaest., D.

\section{Cf. Vendita cit. (nota 14 sopra), p. 451}

22. Cf. Knütel, Augusto Teixeira de Freitas e il "periculum" nella compravendita, in Augusto Teixeira de Freitas e il diritto latinoamericano, Padova, 1988, p. 489.

La situazione del diritto classico riguardo il nostro tema corrisponderebbe dunque in modo parziale, e cioè in certi gruppi di passi ed in determinate fattispecie, all'ordinamento tedesco sia del periodo precedente clie seguente la codificazione. Riguardo a quest'ultimo, il BGB infatti tratta della questione specialmente nel suo $\$ 446$ [ 1: il venditore, come regola generale, sopporta i rischi del perimento fortuito o deterioramento casuale fino alta consegna della cosa venduta al compratore. Da sottolinearsi che basta per la trasmissione dei rischi la semplice "Ubergabe" o consegna, e cioè non si mostra necessario per l'incidenza della regola il trasferimento effettivo della proprietà (valendo dunque anche in casi di possessio o di sola detenzione): cf. Haymann, Textkrit. // cit. (nota 6 sopra), p.149, e Knütel, ibidem. Questa regola generale si riferisce specialmente alle cose mobili. Per quanto riguarda invece gli immobili e le navi marittime, i rischi, secondo lo stesso $\$ 446 \mathrm{II}$, sono addossati al compratore già a partire dalla registrazione nel "Grundbuch" I $\$ \S 324$ ll e 447 infine stabiliscono eccezzioni riguardanti i casi di "Versendungstiauf", o vendita per corrispondenza, e di "Annahmeverzug", e cioè di mora d'accettazione del compratore al momento del perimento fortuito della cosa venduta.

Questa regolamentazione è seguita pure da altri moderni ordinamenti, tra cui l'austriaco ABGB $(\$ \S 1.064,1.048,1.049$ e 1.051$)$ e il Codice Civile brasiliano (artt. 865, 866, 868 e 1.127).

23. Paul. 3 Alfeni epitomarum, D. 18,6,13: "Lectos emptos aedilis, cum in via publica positi essent, concidit: si traditi essent emptori aut per eum stetisset quo minus traderentur, emptoris periculum esse placet...

Paul. 3 epitomarum Alfeni, D. 18,6,15 pr.-1: "Quod si neque traditi essent neque emptor in mora fuisset quo minus traderentur, venditoris periculum erit. l. Materia empta si furto perisset, postquam tradita esset, emptoris esse periculo respondit, si minus venditoris: videri autem trabes traditas, quas emptor signasset"

Su questo gruppi di passi, cf. in speciale: Jhering, Gesammmte Aufsätze aus den Jahrbüchern für die Dogmatik des heutigen römischen und deutschen Privatrechts I, Jena, 1881 [=Aalen, 1981], pp. 469 ss.; Puntschart, Fundamentale Rechtsverhältnisse des römischen Privatrechts, Innsbruck, 1885, pp. 234 ss. (con resoconto della discussione della pandettistica); Haymann, Textkritik II cit., (nota 6 sopra), pp. 73 ss.; Rabel, Gefahrtragung cit. (nota 17 sopra), pp. 552 ss; Appleton, Les risques dans la 
$19,2,33,{ }^{24}$ riguardanti i famosi casi del fundus publicatus, dei lecti empti distrutti dall'edile e della materia empta rubata: nonostante l'erculeo sforzo della communis opinio --non soltanto la tradizionale ma anche l'attuale--, tutti i tentative di armonizzare la soluzione di questo frammento con il (preteso) "principio" periculum est emptoris sono a nostro avviso interamente falliti, ${ }^{25}$ in queste fattispecie semplicemente, è stata la soluzione contraria -periculum est venditoris-, fondata (abbastanza chiaramente nei testi di Paolo, meno in quello di Africano) sul principio della traditio, ad essere accolta. Il fatto che la dottrina abbia esageratamente

vente et les fausses interpolations, in RH 5 (1926), pp. 410 ss.; Hoetink, "Periculum est emptoris", Haarlen, 1928, pp. 126 ss.; Beseler, Romanistische Studien. in TR 8 (1828), pp. 297 ss.; Seckel-Levy, Die Gefahrtragung cit. (nota 8 sopra), pp. 248 ss.: Krückmann, "Periculum emptoris", in ZSS 60 (1940), pp: 65 ss.; Arangio-Ruiz, La compravendira II cit. (nota 4 sopra), pp. 234 ss.; Sargenti, Problemi della responsabilità contrattuale, in SDHI 20 (1954), pp. 248 ss.; Mayer-Maly, Haftung aus miete nach Staatsunrecht, in ZSS 74 (1957) pp. 365 ss.; Provera, Sul problema del rischio contrattuale nel diritto romano, in Studi in onore di Emilio Betti III, Milano, 1962, pp. 708 ss.; 253; Miquel, "Periculum" cit. (nota 2 sopra), pp. 143 ss.; Benöhr, Das sogenannte "Synallagma" in den Konsensualkontrakten des klassischen römischen Rechts, Hamburg, 1965, pp. 89 ss.; Kaser, Das römische I cit. (nota 1 sopra), p. 553, n. 70; Peters, "Iuris Professio" in Festgabe Kaser z. 80. Geburtstag, 1986, pp. 231 ss.; e Thielmann, Traditio cit. (nota 11 sopra), pp. 301 ss.

24. Afric. 8 quaestionum, D. 19,2,33: "Si fundus quem mihi locaveris publicatus sit, teneri te actione ex conducto, ut mihi frui liceat, quamvis per te non stet, quo minus id praestes: quemadmodum, inquit, si insulam aedificandam locasses et solum corruisset, nihilo minus teneberis. 'Nam et si vendideris mihi fundum isque priusquam vacuus traderetur publicatus fuerit, tenearis ex empto: quod hactenus verum erit, ut pretium restituas, non ut etiam id praestes, si quid pluris mea intersit cum vacuum mihi tradi'. Similiter igitur et circa conductionem servandum puto. Ut mercedem quam praestiterim restituas, eius scilicet temporis, quo fruitus non fuerim, nec ultra actione ex conducto praestare cogeris. Nam et si colonus tuus fundo frui a te aut ab eo prohibetur, quem tu prohibere ne id faciat possis, tantum ei praestabis, quanti eius interfuerit frui, in quo etiam lucrum eius continebitur: sin vero ab eo interpellabitur. quem tu prohibere propter vim maiorem aut potentiam eius non poteris, nihil amplitus ei quam mercedem remittere aut reddere debebis... Su questo testo, cf. specialmente: Bechman, System des Kaufs nach gemeinem Recht 1II.1, Leipzig, 1905 , pp. 166 ss.; Haymann, Textkrit. // cit. (nota 6 sopra), pp. 63 ss.); Appleton, (nota 23 sopra), pp. 405 ss.; Hoetink, cit. (nota 23 sopra), pp. 104 ss.; Seckel-Levy, Die Gefahrtragung cit. (nota 8 sopra), pp. 219 ss.; Haymann, Zur Klassizitäı cit. (nota 6 sopra), p. 410, n. 2; Krïckmann, (nota 23 sopra), pp. 74 ss.; Arangio-Ruiz, La compravendita // cit. (nota 4 sopra), p. 270, n. 1; Kaser, "Periculum locatoris", in ZSS 74 (1957), pp. 177 ss.; Provera, (nota 23 sopra), pp. 2.252 ss.; Benöhr, (nota 23 sopra), pp. 87 ss.; Honsell, Quod interest im "bonae-fidei-ilıdicium" Studien zum römischen Schadensersatzrecht, München, 1968, pp. 122 ss.; Kaser, Das römische / cit., p. 553, n. 72; Wacke, Dig. 19,2,33: Africans Verhältnis zur Julian und die Hafrung für höhere Gewall, in ANRW II 15 (1976), pp. 455 ss.; Ankum, African Dig. 19.2.33: Haftung und Gefahr bei der "publicatio" eines verpachieten oder verkaufen Grundstücks, in ZSS 97 (1980), pp. 157 ss.; Ernst, "Periculum" cit. (nota 10 sopra), pp. 241-242; Molnár, "Periculum emploris" im römischen Recht der klassischen Periode, in Studi in onore di Guarino V, Napoli, 1984, pp. 2.252 ss.; Peters, (nota 23 sopra), pp. 231 ss.; e Thielmann, "Traditio" cit. (nota 11 sopra), pp. 299-301.

25. Nello stesso senso, ma limitatamente a D. 18,6,13 e 15 pr.-1, cf. Talamanca, Vendita cit. (nota 14 sopra), p. 452. 
speculato sui dati di fatto eventualmente presenti in queste fattispecie (specialmente in D. 19,2,33) è da ricondurre non alla mancante "Überlieferung" di "näheren Einzelheiten der tatsächlichen Verhältnisse" come vuole il Thielmann, ${ }^{26}$ bensì alla sopramenzionata infruttifera fatica della communis opinio.

Ed allora, accanto a questi passi (appena riferiti) in tema di periculum rei venditae in cui il principio della traditio si mostra applicato --od almeno pressupposto-- ci sembra da doversi elencare, come si cercherà di dimostrare nello studio esegetico che si segue, anche i passi chiavi in tema di periculum dotis aestimatae, e cioè Ulp. 34 [ad ed.]<ad Sab.>, D. 23,3,14, e Pomp. 14 ad Sab., D. $23,3,15$.

III - Aspetti generali del problema del Periculum Dotis Aestimatae

III. 1 La dos Aestimata: Nozione

La cosiddetta dos aestimata (chiamata anche dalla dottrina aestimatio dotis oppure res aestimata in dotem data) é notoriamente un modo particolare, piuttosto complicato, di costituzione di dote. ${ }^{27}$

Per mezzo della dos aestimata venditionis causa, ${ }^{28}$ la fidanzata si obbliga a trasferire certi beni a titolo di dote, convenendo con il futuro marito che soluto matrimonio dovranno essere restituiti non gli stessi oggetti, bensì la loro aestimatio, e cioè il loro prezzo di stima, precendentemente accertato. Tale convenzione si presenta nella compilazione configurata giuridicamente come una compravendita, sottomessa alla condizione sospensiva "si matrimonium fuerit seculum": ${ }^{29}$ verificatosi il matrimonio e diventato così efficace il negozio accordato,

26. Traditio cit. (nota 11 sopra), pp. 300-30I.

27. Cf. Santilippo, Corso di diritlo romano. La dote. l, Catania, s.d., pp. 78 ss.

28. In dottrina si è soliti distinguere tra la dos aestimata venditionis causa e taxationis causa, i cui effetti giuridici sono in parte diversi, giacchè nel primo caso si accorda, soluto matrimonio, la restituzione unicamente del valore di stima, mentre nel secondo si accorda la possibilità al marito di scegliere tra la aestimatio o gli stessi bene dotali. Ci tratterremo sulla prima figura, giacchè è quella che presenta importanza per quanto riguarda il problema collegato del periculum nella compravendita. Sulla aestimatio dotis taxationis causa (con le sue diverse "Erscheinungsformen"), il cui numero di passi tra l'altro è --in confronto alla prima figura-- molto più ridotto, cf., in speciale, Burdese, "Aestimatio dotis", in Studi Betti II. Milano, 1962, pp. I 70 ss., e Streicher, "Periculum dotis" Studien zum dotalrechtlichen Haftungssystem im klassichen römischen Recht, Berlin, 1973, pp. 20 ss.

29. Cf. Ulp., 34 ad Sab., D. 23,3,10,4. 
la moglie-venditore è tenuta a trasferire la proprietà dei cespiti, mentre il maritocompratore si obbliga a pagarne il prezzo (o valore di stima), il quale però è trattenuto da lui a titolo di dote, costituendo quindi l'oggetto della restituzione soluto matrimonio. $^{30}$

Si discute però in dottrina da un lato 1) se siano stati i classici ad assimilare la aestimatio dotis alla compravendita, e dall'altro, ammessa la classicità di tale assimilazione, 2) entro quali limiti siano stati applicati a questo speciale modo di costituzione di dote il regime o le regole tipiche della compravendita.

Quanto al primo punto l'ampia maggioranza degli autori -specialmente Beseler ${ }^{31}$ e Volterra, ${ }^{32}$ così come (sotto certi aspetti) Lauria, ${ }^{33}$ Miquel, ${ }^{34}$ Cannata $^{35}$ e $\mathrm{Kaser}^{36}$-- è solita ritenere classica l'equiparazione aestimatio dotis=venditio; Albertario, ${ }^{37}$ Erhardt $^{38}$ e Calonge, ${ }^{39}$ la giudicano invece giustinianea, ${ }^{40}$ mentre altri autori, come Wolff $^{41}$ e Burdese, ${ }^{42}$ assumono una posizione intermedia, ravvisando nel diritto classico la presenza di spunti che sarebbero serviti di base posteriormente ai giustinianei nella costruzione teoricodogmatica (Wolff), ${ }^{43}$ oppure nell'affermazione legislativa (Burdese) ${ }^{44}$

30. Cf. Sanfilippo, La dote cit. (nota 27 sopra), idem, ibidem.

31. Miscellanea, in ZSS 45 (1925), pp. 257 ss.

32. In tema di "aestimatio dotis", in RIL 66 (1933), pp. 1.014 ss. [= Scritti giuridici I, Napoli, 1991, pp. 429 ss.].

33. Matrimonio-Dote in diritto romano, Napoli, 1952, pp. 140 ss.

34. "Periculum" cit. (nota 2 sopra), pp. 154 ss.

35. Dote (diritto romano), in ED 14 (1965), p. 5, n. 25.

36. Das römische Privatrecht I cit. (nota I sopra), p. 340.

37. "Subtilitas legum" $e$ "moderamen naturalis iuris" nel diritto dotale romano giustinianeo, in RIL 58 (1925), p. 831 [= Studi I, Milano, 1933, p. 394], e Interpolazioni in D. 20, 4, 9, 3 (Appunti sulla "aestimatio dotis", in RIL 59 (1926), pp. 5-6 (estratto) [=Studi I, Milano, 1933, pp. 405-406].

38. "Iusta causa traditionis", Berlin-Leipzig, 1930, pp. 91 ss.

39. "Aestimatio dotis", in AHDE 35 (1965), pp. 43 ss.

40. Lo Streicher, "Periculum" cit. (nota 28 sopra), pp. 1-2 e 6, pur confinando questa "Streitfrage" riguardante la classicità dell'equiparazione cestimatio=venditio nell'ambito del periculum nella dos aestimata (in speciale nell'esegesi di D. 23,3,10,4-5), la ritiene postclassica: "Dann aber stellt sich die Gleichung aestimatio=venditio jedenfalls hier als nachklassische Zutat dar"

41. Zur Stellung der Frau im klassischen römischen Dotalrecht, in ZSS 53 (1933), pp. 331 ss.

42. "Aestimatio dotis" cit. (nota 28 sopra), pp. 203 ss. 
dell'assimilazione aestimatio dotis=venditio.

Per quanto riguarda il secondo punto, la dottrina quasi unanimemente $^{45}$ eccettuati Beseler e Volterra ${ }^{46}$ - sostiene che non tutte le regole della vendita venivano applicate e cioè che la causa dotale esercitava pure una certa efficacia, con l'impiego in certi casi del regime proprio della dos.

\section{III.2 Il Periculum Dotis Aestimatae: Premesse}

Di fronte a queste grandi incertezze, principalmente intorno ai limiti in cui l'assimilazione aestimatio=venditio sia stata accolta in epoca classica, risulta chiaro che anche la questione del periculum dotis, per quanto concerne specificamente il problema della determinazione dell'esatto momento della trasmissione del rischio riguardante la res aestimata in dotem data al marito (e cioè una possibile influenza, ad esempio, esercitata dall'elemento rappresentato dalla traditio) -punto che più ci interesserà in questo studio-, rimane in aperto. Da ciò deriva per forza che $\mathrm{i}$ risultati fino addesso ottenuti dalla dottrina in tema di periculum dotis (nei limiti appena riferiti) non possono a priori servire né come prova a favore della classicità del principio periculum est emptoris, difesa dalla communis opinio, né contro. Anzi, ammesse le conclusioni generali in tema di periculum dotis raggiunte dallo Streicher, ${ }^{47}$ forse l'unico ${ }^{48}$ autore ad occuparsi

43. L'inquadramento (più teorico che pratico) sistematico della aestimatio dotis nel regime della compravendita, operato dai compilatori, sarebbe stato preparato, secondo Wolff, Zur Stellung cit. (nota 41 sopra), pp. 331 ss., in base alla prassi del classici: "Die von den Späten vorgenommene, mehr theoretisch als praktisch bedeutsame systematische Einordnung in das Kaufrecht wurde aber durch die Praxis der Klassiker vorbereitet", idem, ibidem, p. 332

44. L'assimilazione della aestimatio dotis alla compravendita sarebbe stata invece dai classici costruita, secondo il Burdese, "Aestimatio dotis" cit. (nota 28 sopra), p. 204, "in sede di intepretazione tipica integrativa dell'assetto di interessi divisato dalle parti"

45. Cf, oltre gli autori citati nelle note nn. Il a 18, anche Bonfante, Corso di diritto romano. 1. Diritto di Famiglia, Roma, 1925, pp. 309-310 e Grosso, Ricerche intorno all'elenco classico dei "bonae fidei iudicia" I. "Iudicium rei uxoriae", in RISG 3 (1928), pp. 43 ss. (estratto).

46. Cf. rispettivamente $\mathrm{nn} .31$ e 32 sopra.

47. "Periculum dotis" cit. (nota 28 sopra), pp. 102 ss. Cf. anche le Recensioni di Honsell, in IURA 24 (1973), pp. 330 ss., Lemosse, in RH 52 (1974), pp. 311 ss., e Wacke, Zur Funktion und Gefahrtragung bei der römischen Mitgift, in TR 43 (1975), pp. 241 ss.

48. Cf. le osservazioni in questo senso fatte dallo stesso Streicher nell'introduzione (pp. 1-2) al suo lavoro; si ricordi per l'altro che Honsell, in precedenza, si aveva occupato del tema, limitandolo però al "periculum nominis": cf. Das "periculum nominis" im Dotalrecht, in ZSS 83 (1966), pp. 365-373. 
monograficamente del tema del periculum dotis, e cioè che $\mathrm{i}$ "... Zurechnungskriterien für das periculum dotis sehr vielfältig sein können" -fondandosi in parte anche al di fuori del "Dotalrechts" (come presso le res aestimatae)--, ma ispirati comunque dal melius aequius, dalla bonas fides e dal "Utilitätsprinzip" 49 la questione del periculum dotis viene ad sere impostata esattamente nel modo da noi ritenuto (come si vedrà alla fine) il più giusto in relazione al periculum rei venditae, vale a dire, anche in questo caso presupporre per il diritto classico l'adozione di una "elastische Gebilde", in cui certi elementi, come specialmente la traditio, possono aver svolto un ruolo importante in alcuni casi.

Non ci sembra dunque affatto, come è solita ammettere la communis opinio, ${ }^{50}$ che alla domanda sul preciso momento della trasmissione dei rischi al marito, nell'ambito della dos aestimata venditionis causa, Ulpiano risponda a priori interamente o chiaramente in questo gruppo di passi:

Ulp. 34 ad Sab., D. 23,3,10,4-5: "Si ante matrimonium aestimatae res dotales sunt, haec aestimatio quasi sub condicione est: namque hanc habet condicionem 'si matrimonium fuerit secutum: Secutis igitur nuptiis aestimatio rerum perficitur et fit vera venditio. 5. Inde quaeri potest, si ante nuptias mancipia aestimata deperierint, an mulieris damnum sit, et hoc consequens est dicere: nam cum sit condicionalis venditio, pendente autem condicione mors contigens exstinguat venditionem, consequens est dicere mulieri perisse, quia nondum erat impleta venditio, quia aestimatio venditio est"

Su questo gruppo di passi gravano numerosi sospetti di interpolazione, che riguardano specialmente, nel primo passo (D. 23,3,10,4), l'intera frase "et fit vera venditio" 51 --oppure soltanto l'aggettivo "vera" ${ }^{\prime 52}-$ e, nel secondo

49. Cf. Periculum dotis cit. (nota 28 sopra), pp. 102-103. Il Streicher ritiene che, tra questi criteri di responsabilità, quello della bonas fides sia stato il più significativo.

50. Cf., ad es., Streicher, Periculum dotis cit. (nota 28 sopra), p. 4.

51. Cf, in questo senso, specialmente, Erhardt, "lusta causa" cit. (nota 38 sopra), pp. 93 ss.; Wolff, Zur Stellung cit. (nota 41 sopra), p. 335, n. 2; Burdese, "Aestimatio dotis" cit. (nota 28 sopra), pp. 182 ss.; Streicher, "Periculum dotis" cit. (nota 28 sopra), pp. 4 ss.

52. Cf., in questo senso, specialmente, Volterra, In tema di "aestimatio dotis" cit. (nota 32 sopra), p. 1.015 [=pp. 430] e Sanfilippo, La dote cit. (nota 27 sopra), pp. 79 ss.

Non mancano però autori che lo ritengono interamente genuino: cf., in questo senso, specialmente, Haymann, Textkrit. I/ cit. (nota 6 sopra), p. 85, e Zur klassizität cit. Zur klassizität des "periculum emptoris" in ZSS 48 (1928) (nota 6 sopra), p. 349, n. 1. 
(D.23,3,10,5), il periodo "hoc consequens est...venditionem", 53 nonchè l'inciso finale "quia" rell. 54

La communis opinio comunque, pur riconoscendo i difetti formali, tende a ritenerli sostanzialmente genuini. ${ }^{55}$

Ed ecco l'interpretazione di questi passi di solito proposta dalla communis opinio: $:^{56}$ essendo l'aestimatio una vendita, dovrebbero incidere le stesse regole dell'emptio venditio, quindi periculum est emptoris. Quella però non è una vendita pura bensì condizionale ed il perimento della res avviene prima del matrimonio --cioè anteriormente al verificarsi della condizione-- perciò, prima che la vendita divenga perfecta. Per questa ragione il danno sarebbe a carico della donna, giacchè soltanto "perfecta emptione periculum ad emptorem (cioè marito) respiciet" (Paul. 33 ad ed., D. 18,6,8 pr.).

Partiamo dal pressuposto, come riconosciuto dalla communis opinio, che non tutte le regole della emptio venditio venissero applicate in tema di dos aestimata. Lasciamo dunque da parte la regola "perfecta emptione (=il verificarsi della condizione) periculum ad emptorem respiciet" appena ricordata, partendo dal pressuposto, come si è detto, che in nostro tema sia stato applicato un regime proprio della dos aestimata.

Il testo allora non può a nostro avviso servire come testimonianza dell'accoglimento del "principio" periculum est emptoris, giacchè, come si è già detto sopra, non risponde alla specifica domanda sul preciso momento del passaggio dei rischi al marito. Ulpiano infatti afferma soltanto che, verificatasi la condizione (e

53. Cf. in questo senso, specialmente, Haymann, Textkrit. I/ cit. (nota 6 sopra), pp. 85 ss.; Erhardt, "Iusta causa" cit. (nota 38 sopra), pp. 93 ss; Burdese, "Aestimatio dotis" cit. (nota 28 sopra), pp. 182 ss.; e Calonge, "Aestimatio dotis" cit. (nota 39 sopra), pp. 43 ss.

54. Cf. in questo senso, specialmente, Haymann, Textkrit. II cit. (nota 6 sopra), pp. 85 ss.; Bonfante, Corso I cit. (nota 45 sopra), p. 309, n. 3 (che lo ritiene un glossema, di significato però giusto); Erhardt, "Iusta causa" cit. (nota 38 sopra), pp. 93 ss.; Burdese, "Aestimatio dotis" cit. (nota 28 sopra), pp. 182 ss.; Sanfilippo, La dote cit. (nota 27 sopra), p. 80; Calonge, "Aestimatio dotis" cit. (nota 39 sopra), pp. 43 ss.; e Streicher, "Periculum dolis" cit. (nota 28 sopra), pp. 4 ss.

55. Cf., in questo senso, specialmente Bonfante, Corso / cit. (nota 45 sopra), p. 309, n. 3; Volterra, In tema di "aestimatio dotis" cit. (nota 32 sopra), p. 1.015 [=pp. 430]; Streicher, "Periculum dotis" cit. (nota 28 sopra), p. 6 ("Von den oben gennanten formalen Mängeln abgesehen, ist der Kern der $\$ \S 4,5$ klassischen Ursprungs"); e Wacke, Zur Funktion cit. (nota 47 sopra), p. 250, n. 2. Contro: Erhardt, "Iusta causa" cit. (nota 38 sopra), pp. 93 ss.; Burdese, "Aestimatio dotis" cit. (nota 28 sopra), pp. 182 ss.; e Haymann, Textkrit. I/ cit. (nota 6 sopra), p. 85; Zur Klassizität cit. (nota 6 sopra), p. 349 , n.l (soltanto per il fr. 4).

56. Cf. Sanfilippo, Corso cit. (nota 27 sopra), p. 80. 
cioè la realizzazione delle nozze), il marito passa a sopportare i rischi della cosa oggetto della dos aestimata.

Si tratta semplicemente di una dichiarazione generale del giurista, che centra la sua attenzione sul problema della condicio: questa avvenuta, dice Ulpiano, $\mathrm{i}$ rischi sono trasmessi al marito, come è principio generale in materia di dos aestimata, vale a dire, una regola appartenente al regime proprio della dote estimada (Ulp., 34 ad ed., D. 23,3,10 pr.: "Plerumque interest viri res non esse aestimatas idcirco, ne periculum rerum ad eum pertineat..."). Questa regola differisce da quella dei casi normali di dote --e cioè non aestimata-- (di cose non fungibili), ${ }^{57}$ in cui il periculum rimane a carico della moglie (pur essendo la cosa consegnata nella possessio del marito), e quest'ultimo risponde soltanto per "culpa": "Paulus respondit rebus non aestimatis in dotem datis maritum culpam, non etiam periculum praestare debere" (Frag.Vat. 101).

La dichiarazione di Ulpiano in queste fattispecie dunque non riguarda la questione risultante dall'eventuale consegna o meno della res aestimata in dotem data al marito, e cioè la possibile influenza che un tale elemento --il verificarsi della traditio-- possa avere sull'attribuzione del periculum; non tratta quindi del problema concernente alla determinazione esatta e specifica del momento della trasmissione dei rischi al marito. ${ }^{58}$

Ciò però può essere spiegato: quel problema è trattato da Ulpiano soltanto appunto in 1. 34 [ad ed.] <ad Sab.>, ${ }^{59}$ D.23,3,14. Cosa questa peraltro coerente: ammessa la correzione proposta da Lenel (come infatti è comumente accettato) nella sua Palingenesi, e cioè che il frammento 14 in origine apparteneva al I. 34 dei commentari di Ulpiano ad Sab. e non ad ed., avremmo ambi i passi --

57. Per le cose fungibili invece le fonti sembrano (cf. però nota 58 infra) accogliere la stessa soluzione applicata alla dos aestimata, e cioè periculum est mariti: Gai 11 ad ed. prov.: "Res in dotem datae, quae pondere numero mensura constant, mariti periculo sunt, quia in hoc dantur, th eas maritus ad arbitrium suum distrahat et quandoque soluto matrimonio eiusdem generis et qualitatis alias restituat vel ipse vel heres eius"

58. Da notare che pure Gaio in D. 23,3,42 (cf. nota 57 sopra), trattando del periculum spettante le cose fungibili non aestimatae, non si occupa della determinazione dell'esatto momento in cui i rischi sono trasmessi al marito (cf. in questo senso Miquel, Periculum locatoris cit. [nota 2 sopra], p. 157: "La soluzione data al problema del periculum con riguardo alle cose fungibili non è quindi diversa da quella delle res aestimatae in dotem datae: periculam est mariti. Diverso è soltanto il momento del trapaso del rischio, ma il testo di Gaio tace a questo proposito"). Si potrebbe dunque discutere anche in tema di dos non aestimata di cose fungibili, la possibile influenza della traditio nella specificazione del momento della trasmissione dei rischi al marito.

59. Cf. nota 88 infra. 
D.23,3,14 e D.23,3,10,4-5-- estratti dallo stesso libro 34. Il giurista in questo libro dunque, dopo la sua dichiarazione (o soluzione) generale in tema di periculum res aestimatae, riprodotta nella fattispecie di D.23,3,10,4-5, si occupava di seguito, come risulta da D. 23,3,14, della determinazione del preciso momento della trasmissione dei rischi, e cioè del problema riguardante gli effetti della traditio sulla ripartizione del periculum dotis aestimatae.

L'interpretazione della communis opinio quindi non regge. Si ricordi tra l'altro che, contro tale interpretazione, parte della dottrina --Haymann ${ }^{60}$ e Betti ${ }^{61}$,pur minoritaria, ritiene il testo sostanzialmente interpolato. ${ }^{62}$ Indipendentemente però dalla dimostrazione del carattere spurio o non del testo, c'è tuttavia la nostra interpretazione sopra riferita: Ulpiano, nella sua decisione, afferma soltanto che, non verificatesi le nozze --e cioè la condizione--, il rischio incombe sulla moglie. Il giurista dunque non discute affatto il problema specifico dell'attribuzione del rischio tra il momento della costituzione della dote (e della aestimatio) e l'effettiva consegna della res al marito, non gli interessa cioè la domanda se la cosa è stata o no consegnata (vale a dire, il ruolo che potrebbe avere la traditio). Anzi, si potrebbe dire, in questo ordine di idee, che il verificarsi o non della consegna della res al marito non cambierebbe la soluzione finale: nella ratio decidendi o nel ragionamento di Ulpiano conta soltanto il fatto che il perimento si è verificato pendente condicione, e cioè prima delle nozze, il che escluderebbe qualsiasi responsabilità del marito, sia egli in possesso della cosa o non. Vale a dire, con altre parole, non perchè "perfecta emptione (=il verificarsi della condizione) periculum ad emptorem respiciet" (D. 18,6,8 pr. - regime proprio della vendita), ma perchè "res aestimatas esse, periculum rerum ad virum pertineat" (ad arg. D. 23,3,10 pr.).

D'altra parte, non si deve escludere, come già proposto da Ehrhardt, ${ }^{63}$ che la res fosse stata già consegnata al marito. In questo senso, la decisione di

60. Textkrit. II cit. (nota 6 sopra), pp. 85 ss., e Zur Klassizität cit. (nota 6 sopra), p. 416, n. 3.

61. "Periculum" cit. (nota 7 sopra), p. 149.

62. Anche il Burdese, "Aestimatio dotis" cit. (nota 28 sopra), pp. 182 ss., pur ritenendo classico il principio periculum est emptoris --ibidem, pp. 187-188, n. 74--, condanna, ma per ragioni diverse da quelle di Haymann e Betti, grande parte del frammento, ricostruendo il testo originale con l'eliminazione di qualsiasi accenno alla compravendita: spurie sarebbero le frasi "et fit vera venditio", "hoc consequens est...venditionem" e la chiusa "quia" rell., il cui richiamo sarebbe interamente inutile nella fattispecie.

63. "Iusta causa traditionis" cit. (nota 38 sopra), p. 93: "Vor Eheabschluß sind aestimata mancipia, die dem Bräutigam zur dos übergeben waren, gestorben" 
Ulpiano avrebbe ancora più ragione di essere: egli vorrebbe sottolineare che, pur essendo la cosa già in possesso del marito, il rischio andrebbe alla donna qualora la condizione non si fosse verificata. ${ }^{64}$

Resterebbe finalmente da discutere un ultimo punto a proposito di D. $23,3,10,4-5$. Osserva Streicher nella sua esegesi a questo gruppo di passi che, al contrario che nella compravendita, non ci sono nelle fonti indizi in base ai quali si possa dedurre che la moglie doveva sopportare, fino alla consegna del bene dotale, il periculum custodiae. ${ }^{65}$ Da questa osservazione si può a nostro avviso dedurre un ulteriore argomento contro la communis opinio: ammessa come classica l'equiparazione aestimatio $=$ venditio, $\mathrm{i}$ pochi autori che rifiutano una responsabilità per custodia del venditore (sostenuta specialmente da Seckel) potrebbero richiamare questa possibile corrispondente inesistenza di tale responsabilità della moglie nella dos aestimata come un indizio a favore della loro tesi (anche se si potrà sempre contro argomentare con la non completa applicazione delle regole della compravendita in materia di dos aestimata, secondo quanto ritenuto dalla communis opinio). ${ }^{66}$ Dall'altra parte, ammessa ancora come probabile la non responsabilità della moglie per custodia prima della consegna del bene dotale al marito, la situazione di quest'ultimo diventerebbe molto più gravosa di quella del compratore (se si accetti evidentemente la ricostruzione del Seckel). Ciò comunque potrebbe servire come argomento contro quelli, come Streicher, che ritengono --in materia di dos aestimata contrariamente a quanto da noi sostenuto-- non doversi ammettere importanza alla traditio per quanto riguarda l'attribuzione del periculum, giacchè è

64. Contro Ehrhardt si manifesta specialmente Streicher, "Periculum dotis" cit. (nota 28 sopra), p. 5, n. 17: "Seine Annahme, es handle sich um aestimata mancipia, die bereits übergeben waren, findet im Text keine Stütze" Comunque, la stessa esistenza di letture diverse per quanto riguarda il verificarsi o non della traditio in questa fattispecie, è già una dimostrazione della completa mancanza di riferimento nel testo al problema della eventuale consegna, il che si spiega, come già detto, con la scarsa importanza di tale elemento nel ragionamento di Ulpiano.

65. Cf. "Periculum dotis" cit. (nota 28 sopra), p. 6 e n. 22. L'autore sembra addiritura rifiutare una responsabilità per custodia della moglie in tema di dote: "In deutlichen Unterschied zum Kaufrecht ist den Quellen indes kein Hinweis zu entnehmen, da $\beta$ die Frau bis zur Übergabe der Sache das periculum custodiae hätte tragen müssen" (p. 6); ed ancora: "...aber im Gegensatz zum Kaufrecht muß die Frau in keinem Fall vor Übergabe der Sache dem Mann für etwaigen Untergang Ersatz leisten" (p. 6, n. 22).

Contro si esprime il Wacke, Zur Funktion cit. (nota 47 sopra), p. 251, nella recensione al lavoro di Streicher: "daß die Frau bis zur Übergabe freilich wie beim echten Kauf für custodia verantwortlich war, ist zwar nicht zu belegen, aber doch wahrscheinlich" Cf. nota 92 infra.

66. Cf. fls. 12 sopra. 
ammettere che i classici avessero così sovraccaricato la posizione giuridica del marito, facendolo rispondere per ogni tipo di rischio già a partire dal momento della conclusione del patto di aestimatio.

\section{L'esegesi dei passi "Clavis Huius Materiae"}

Ed ecco quindi i due passi fondamentali normalmente richiamati in tema di rapporti tra periculum rei venditae e periculum dotis aestimatae, i quali a parere della communis opinio (come si è detto già sopra), ${ }^{67}$ rappresenterebbero testimonianze abbastanza esplicite e fededegne del principio che addossa al compratore i rischi già a partire dal momento di conclusione del contratto:

D. 23,3,14 (Ulpiano, 34 ad ed.): "Si rem aestimatam mulier in dotem dederit, deinde ea moram faciente in traditione in rerum natura esse desierit, actionem eam habere non puto".

D. 23,3,15 (Pomponio, 14 ad Sab.): "Quod si per eam non stetisset, perinde pretium aufert ac si tradidisset, quia quod evenit emptoris periculo est"

Se la moglie, dice Ulpiano nel primo passo, abbia dato in dote una cosa estimata e, in seguito, essendo ella in mora nella sua consegna, la cosa cessasse di esistere in natura, e cioè perisse, la moglie non avrebbe azione $<$ per la restituzione della dote $>$ Se però, dichiara Pomponio nel frammento seguente, ciò non fosse dipeso da lei, essa potrebbe pretendere il prezzo come se avesse fatto la consegna, giacchè ciò che avviene è a carico del compratore, che sopporta i rischi.

I giuristi quindi, secondo l'interpretazione della communis opinio, avrebbero differenziato, in caso di perimento fortuito della res aestimata prima della traditio, due situazioni: ${ }^{68}$ 1) se la moglie fosse in mora, non avrebbe contro il marito azione allo scopo di esigere la restituzione della aestimatio; 2) se invece non fosse in mora, si applicherebbe il principio periculum est emptoris ed ella, in contrapartita, avrebbe diritto alla restituzione del prezzo.

In tema dunque di periculum dotis aestimatae, conclude la communis

67. Cf. fls. 3 e nota 4 sopra.

68. Cf. in questo senso Arangio-Ruiz, La compravendita II cit. (nota 4 sopra), p. 266. 
opinio, ${ }^{69}$ come principio generale si dovrebbe ammettere che il marito doveva sopportare il periculum già a partire dalla realizzazione delle nozze e cioè indipendentemente dal vericarsi o non della traditio, la quale non avrebbe alcuna importanza in tema di ripartizione dei rischi, esattamente come nella compravendita (periculum est emptoris).

Procediamo all'esegesi separata dei due passi. Tale metodo, il più giusto al meno dal punto di vista del diritto classico, ci sembra infatti preferibile a quello della dottrina dominante che suole interpretarli insieme.

\section{IV.1 L'esegesi di Ulp. 34 ad ed., D. 23,3,14}

Ecco in poche parole il contenuto della decisione di Ulpiano: $:^{70}$ la exmoglie (=venditore), dopo la dissoluzione del matrimonio, non può esigere dall'exmarito (=compratore) la aestimatio (=prezzo di vendita) del bene dotale, quando il suo perimento fortuito si sia verificato ${ }^{71}$ mentre lei si trovava in mora nella consegna della cosa.

\section{IV.1.1 Difetti formali}

Il testo è ritenuto formalmente rimaneggiato dalla quasi totalità della dottrina. $^{72}$ Ecco le obiezioni:

a) Se la moglie, come si dichiara nel testo, ha già proceduto alla consegna della dote estimata --"...in dotem dederit..."--, è inammissibile e contradditorio che in seguito, si trovi in mora nella consegna --"... deinde ea moram

69. Cf. in questo senso, da ultimo, Streicher, Periculum dotis cit. (nota 28 sopra), p. 7: "Ob die Sachen übergeben waren oder nicht, spielt keine Rolle, wie der Schluß aus D. 23,3,14 und 15 ergibt. Als Ergebnis können wir somit festhalten: ...b) Nach Eheschließung geht das periculum ganz auf den Mann über, ob die Sachen übergehen sind oder nicht"

70. Cf. Seckel-Levy, Die Gefahrtragung cit. (nota 8 sopra), pp. 260-261.

71. Si pressuppone evidentemente, come già precisava Bas. 29,1,10, sch. e notava la Glossa ad h.l., che il perimento della dos aestimata sia avvenuto dopo il matrimonio:in caso contrario, osserva Grosso, Ricerche cit. (nota 45 sopra), p. 43, n. 4, seguito da Burdese, "Aestimatio dotis" cit. (nota 28 sopra), p. 186, n. 71, tale circostanza, data la sua importanza, non sarebbe stata taciuta.

72. L'unico autore a non presentare obiezionj nemmeno formali contra il passo è Miquel, "Periculum locatoris" cit. (nota 2 sopra), p. 155, che lo ritiene, insieme al passo di Pomponio,
genuino. 
faciente in traditione...". Ulpiano dunque, in origine, avrebbe probabilmente trattato o di un caso di dotis dictio, come proposto per primo da Hotomanus, ${ }^{73}$ seguito da Seckel-Levy, ${ }^{74}$ Arangio-Ruiz, ${ }^{75}$ Betti, ${ }^{76}$ e Calonge, ${ }^{77}$ oppure di una mancipatio non accompagnata dalla effettiva e contestuale consegna, come ritenuto da Beseler ${ }^{78} \mathrm{e}$ Kàser. ${ }^{79}$ Più verosimile ci sembra quest'ultima ipotesi, e cioè una mancipatio che probabilmente ha avuto per oggetto un servus, come proposto dalla Glossa. ${ }^{80}$ Insostenibile comunque è l'argomento di cui si serve il Calonge, ${ }^{81}$ per rifiutare l'ipotesi della mancipatio, e cioè che questa proposta potrebbe essere accettabile soltanto "si se tratara de fundus o servus, pero esto no es posible porque el fundo no perece y el esclavo se 'muere'" Ci pare però chiaro ed indiscutibile che l'espressione latina in rerum natura esse desierit, volendo significare "mancare al mondo, non essere più nel mondo", ${ }^{82}$ possa anche essere impiegato ad indicare la morte, come appunto impiegata in Scev. 21 dig., D. 36,1,80,10 (a contrariis), Pomp. 5 ad Quintum Mucium, D. 36,2,22 pr. e Paolo 41 ad ed., D. 37,6,2 pr. Infine, all'osservazione secondo cui un fondo non può "perecer" si può ribattere menzionando certi casi di alluvio, in cui e.g. un terreno è interamente occupato dalle acque di un fiume (Inst. 3,23,3), ${ }^{83}$ il quale (terreno) appunto --si può dire letteralmente-- "mancherà nella natura"

In ognuno dei due casi ${ }^{84}$ comunque, i compilatori avrebbero eliminato

73. Opp. 1,1, p. 879.

74. Die Gefahrtragung cit. (nota 8 sopra), pp. 260 ss.

75. La compravendita II cit. (nota 4 sopra), p. 266 e n. 3.

76. "Periculum" cit. (nota 7 sopra), p. 149.

77. "Aestimatio dotis" cit. (nota 39 sopra), pp. 20 ss.

78. Romanistische Studien cit. (nota 23 sopra), p. 305. Il Beseler elimina anche la questione della mora, ritenendo spuria l'espressione "moram faciente in traditione" (da essere sostituita secondo l'autore da "res ante traditionem").

79. Die Wirkungen der "dotis dictio", in SDHI 17 (1951), p. 176, n. 25.

80. Cf. Glossa ad h.l., ed. Lugduni, 1593

81. "Aetimatio dotis" cit. (nota 39 sopra), p. 21

82. Cf. il vocabulo natura -"b)B"- nel vocabulario di Heumann-Seckel, Handlexikon zu den Quellen des römischen Rechts, 11 ed., Graz, 1971.

83. Inst. 3,23,3 ("...aut fundus vi fluminis totus vel aliqua ex parte ablatus sit..."): caso citato da Giustiniano appunto come un esempio di periculum che deve essere sopportato dal compratore.

84. Il passo dunque, a seconda delle proposte riferite, potrebbe essere cosi ricostruito: "Si rem 
il riferimento ad istituti già in disuso al tempo di Giustiniano. ${ }^{85}$

b) Manca il soggetto per "desierit" 86

c) Non c'è la qualifica del tipo di actio, anche se sembra logico che si tratti di una actio rei uxoriae. ${ }^{87}$

d) Il passo di Ulipiano sarebbe stato tratto dal I. 34 ad Sab., e non ad $e d$, come ci è stato trasmesso dalla Compilazione. Tale ricostruzione, proposta da Lenel nella sua Palingenesi, è (quasi) ${ }^{88}$ unanimente accettata come probabile dalla dottrina.

Nonostante questi difetti formali, il passo, tranne poche voci in contrario, ${ }^{89}$ è giudicato --a nostro parere con ragione-- sostanzialmente genuino dall'ampia maggioranza degli autori. ${ }^{90}$

aestimatam mulier in [dotem dederit] <doti dixerit $>$ " (o analogamente [dederit] $<$ dixerit $>$, d'accordo con Seckel-Levy) oppure "Si rem aestimatam mulier in dotem <mancipio $>$ dederit"

85. Cf. in questo senso il Burdese, "Aestimatio dotis" cit. (nota 28 sopra), pp. 185-186, il quale, pur ammettendo il rimaneggiamento del testo in questo punto, non prende posizione su queste due ipotesi.

86. Cf. in questo senso, specialmente, Beseler, Romanistische Studien cit. (nota 23 sopra), p. 305 , Seckel-Levy, Die Gefahrtragung cit. (nota 8 sopra), p. 260 (che suppongono che il soggetto della frase nel testo originale fosse rappresentato dal bene dotale specificamente determinato, il quale sarebbe stato parimenti sostituito dai compilatori nella parte iniziale del passo, con il vocabulo generico "rem"), Burdese, "Aestimatio dotis" cit. (nota 28 sopra), pp. 185-186, e finalmente Streicher, "Aestimatio dotis" cit. (nota 28 sopra), p. 10.

87. Cf. in questo senso, specialmente, Burdese, "Aestimatio dotis" cit. (nota 28 sopra), pp. 185186, e Streicher, "Periculum dotis" cit. (nota 28 sopra), p. 10: "weniger anstößig erscheint die mangelnde Determinierung der actio als actio rei uxoriae. Daß die Dotalklage gemeint ist, vesteht sich von selbst"

88. Contro si esprime soltanto il Calonge, "Aestimatio dotis" cit. (nota 39 sopra), p. 19, n. 40: ":.a rectificación de Lenel, comúnmente aceptada, puede que sea válida, pero ante la carencia de una mayor seguridad preferimos suponer que se trate de un comentario al Edicto. Razones tampoco faltan para suponerlo así: Lenel inicia in su Palingenesia el libro 34 ad ed. de Ulpiano bajo el título De re uxoria, donde podría tener cabida nuestro texto, máxime si se tiene present que el núm. 967 (D. $22,1,21$ y D. 22,1,23) face referencia a qué debemos entender por mora" L'argomentazione non ci pare convincente. La ricostruzione di Lenel s'inquadra perfettamente nel probabile contesto originale dell'opera di Sabino, come deducibile anche degli altri commentari ad Sab.

89. Cf. in speciale Haymann, Textkrit. // cit. (nota 6 sopra), p. I3I, che lo ritiene interamente spurio (o, con le sue parole, "heillos zerrütet").

90. Cf. Arangio-Ruiz, La compravendita // cit. (nota 4 sopra), p. 266 e n. 3; Betti, "Periculum" cit. (nota 7 sopra), p. 149; Kaser, Die Wirkungen cit. (nota 79 sopra), p. 176, n. 25; Burdese, "Aestimatio dotis" cit. (nota 28 sopra), pp. 185-186; Grosso, Ricerche cit. (nota 45 sopra), p. 43, n. 4; Miquel, "Periculum locatoris" cit. (nota 2 sopra); e Streicher, "Periculum dotis cit. (nota 28 sopra), pp. 9-10. 


\section{IV.1.2 L'argumentum e contrario}

La forza probante di questo passo in favore della classicità del principio periculum est emptoris si fonda, secondo la communis opinio, su un argumentum e contrario: dalle parole di Ulpiano cioè si dedurrebbe a contrariis che, non essendo la moglie in mora ed essendo avvenuto il perimento fortuito prima della consegna della res aestimata, le competerebbe l'azione per la restituzione del prezzo o aestimatio, e cioè, pur non essendosi verificata tradizione, il danno andrebbe a carico dell'ex-marito, che dovrebbe dunque sopportare il periculum già a partire dalla realizzazione delle nozze, e cioè prima della consegna, esattamente come il compratore.

Contro questa deduzione per argumentum e contrario si possono però opporre due considerazioni.

Da un lato, si deve osservare che il risultato a contrariis ottenuto, e cioè che in caso di inesistenza di mora il danno sarebbe a carico del marito, non è evidentemente dichiarato espressamente dal giurista, bensi soltanto dedotto. Poichè è noto che le decisioni dei giuristi classici dipendevano enormemente dalle peculiarità di ciascuna fattispecie, le deduzioni per argumentum e contrario per quanto riguarda il diritto classico devono sempre essere accolte con molte riserve.

D'altra parte, ammesso soltanto per ipotesi il ragionamento per argumentum e contrario, come proposto dalla communis opinio, non si potrebbe concludere in modo assoluto che, al momento del perimento della cosa, questa ancora non fosse stata consegnata. In altre parole: il fatto (dedotto a contrariis) di "non essere in mora nella tradizione" (=non "ea moram faciente in traditione") non significa necessariamente che "la cosa ancora non è stata consegnata, ma la moglie è ancora in tempo per farlo"; "non essere in mora" insomma potrebbe anche perfettamente indicare che "la cosa già è stata tempestivamente consegnata dalla moglie" (la quale appunto perciò non si troverebbe più in mora o ritardo). Colui che, per ipotesi, ha già consegnato la cosa al momento giusto non si trova in mora; così anche chi non l'ha consegnata, ma è ancora in tempo di farlo. Le due situazioni possono corrrispondere all'affermazione (dedotta a contrariis dalla communis opinio) "non essere in mora"

Questa osservazione potrebbe dunque servire addirittura contro la communis opinio e cioè a favore della tesi che accentua nel nostro tema il ruolo fondamentale svolto dal principio della traditio: essendo la cosa dotale 
tempestivamente consegnata (vale a dire, non essendo la moglie in mora), e verificatosi il perimento fortuito, la ex-moglie potrebbe esigere la restituzione della dote (o meglio, della sua aestimatio); il danno quindi sarebbe a carico del marito, ma la cosa estimata già si trovava nella sua possessio.

IV.1.3 - La mora della moglie: significato ed effetto

Ammesso per ipotesi, come vuole la communis opinio, che in tema di periculum dotis aestimatae il marito sopportasse i rischi già a partire delle nozze, indipendentemente dal verificarsi della traditio, si potrebbe allora chiedere perchè mai Ulpiano, in D. $23,3,14$, addossa il periculum alla moglie in mora nella consegna della cosa estimata? In altre parole: rispondendo il marito già prima della traditio, una eventuale mora della moglie nella consegna non dovrebbe --almeno in termini logici-- svolgere nessun importante ruolo. Ulpiano quindi, a rigore di logica, non avrebbe bisogno di preocuparsi specialmente di quel ritardo della moglie, giacchè la mancanza o non della traditio non avrebbe influenza sulla regola di ripartizione dei rischi, sopportati dal marito.

A questo dubbio la communis opinio suole rispondere richiamando il principio dell'equità: non sembrerebbe giusto ad Ulpiano (continuare ad) attribuire al marito il periculum, quando la moglie fosse in mora nella traditio. "Ulpian", riassume o Streicher," "will nicht die Grundregel des periculum viri bei res aestimatae anwenden, sondern offenbar aus Billigkeitsgründen davon abweichen"

Questa risposta, anche se convincente ad un primo sguardo, non ci sembra però del tutto decisiva. Resta l'apparente ilogicità di una soluzione che, in un primo momento (e cioè a partire dal matrimonio), esonera la moglie dal periculum, in seguito (e cioè in caso di mora) glielo addossa, ed alla fine (e cioè consegnata la cosa) la libera nuovamente. Più coerente si mostrerebbe un sistema che attribuendo all'inizio un certo grado di responsabilità alla moglie, l'aumentasse o sovracaricasse quando essa si trovasse in mora nella consegna. ${ }^{22}$

91. "Periculum dotis" cit. (nota 28 sopra), p. 10.

92. Questa ilogicità --ragionandosi ancora dentro il quadro di ricostruzione storica proposto dalla communis opinio (vale a dire, periculum est emptoris=mariti)--, sparirebbe se si pensasse ad una eventuale responsabilità per custodia della moglie fino alla consegna: realizzate le nozze, essa passava a rispondere soltanto per vis minor (ed il marito per qualsiasi altro tipo di periculum), e di seguito, trovandosi in mora nella traditio, vedrebbe il suo grado di responsabilità aumentare, passando ad sopportare anche la vis maior. 
Si potrebbe certo ribattere che la nostra soluzione --e cioè il sistema opposto-- sarebbe altrettanto illogica: la moglie, prima della traditio, sopportava il periculum, e di seguito, incorrendo nella mora, continuerebbe a sopportarlo, non verificandosi alcun cambiamento.

Questo argomento sarebbe inoppugnabile se non si riuscisse a trovare un'altra giustificazione alla decisione di Ulpiano, che attribuisce il periculum alla moglie in caso di mora, diversa da quella (e cioè, motivi d'equità) della communis opinio. Ciò ci pare possibile, come adesso cercheremo di dimostrare.

Partiamo da una osservazione fatta da Grosso: ${ }^{93}$ in vista del principio, più volte ripetuto dalle fonti, per cui la c.d. aestimatio dotis venditionis causa equivale alla emptio venditio, si dovrebbe teoricamente per conseguenza --osserva l'autore-- ritenere momenti distinti la compravendita e la costituzione di dote, ciò che non è fatto; ${ }^{94}$ a rigor di logica dunque, continua Grosso, la separazione fra la vendita e la dote avrebbe portato, nel passo di Ulpiano (D. 23,3,14), "all'esperimento separato delle due azioni, ex empto del marito contro la moglie, rei uxoriae della moglie verso il marito" ${ }^{95}$

Una eventuale responsabilità per custodia della moglie in casi di dos aestimata non è però, secondo Streicher, "Periculum dotis" cit. (nota 28 sopra) --come già abbiamo visto (cf. pp. 16-17 sopra, nota 65)-- attestata (oppure è esclusa) nelle nostre fonti.

93. Ricerche cit. (nota 45 sopra), pp. 43 ss.

94. Quel principio dunque --afferma ancora Grosso, Ricerche cit. (nota 45 sopra), pp. 43 ss., seguendo in questo punto la communis opinio (cf. tls. 12 n. 45 sopra)--, non avrebbe trovato esatta applicazione, giacchè, nelle soluzioni di casi pratici, anche se talora le regole della emptio venditio venissero applicate, si teneva sempre presente che la vendita era avvenuta a causa della costituzione della dote. In poche parole: la separazione teoricamente necessaria tra la vendita e la dote non era affatto rispettata, per cui, nella risoluzione dei casi pratici, venivano mescolate le regole (e le soluzioni) della dos e della emptio venditio.

95. Cf. Ricerche cit. (nota 45 sopra), pp. 43-44.

Contro Grosso si manifesta Kaser, Die Rechtsgrundlage der "actio rei uxoriae", in Rida 2 (1949), p. 525, n. 67 ("Zu Ulp. D. 23,3,14 behaupt Grosso 43f., die Anwendung der Kaufregeln auf die dos aestimata hätte hier dazu führen müssen, daß der Mann gegen die Frau die actio empti, die Frau gegen den Mann die a. rei uxoriae erhielt. Aber das triftt nicht zu: Ist die Frau im Leistungsverzug, dann ist die Gefahr noch nicht auf den Mann übergegangen [...] und wird er deshalb durch zufälligen Untergang der Sachen befreit"): mentre la moglie era in mora, il rischio non sarebbe stato ancora trasmesso al marito e quest'ultimo quindi non sarebbe stato responsabile per il perimento fortuito della cosa, vale a dire --possiamo aggiungere-- egli non potrebbe essere condanato nella actio rei uxoriae.

D'accordo invece il Burdese, "Aestimatio dotis" cit. (nota 28 sopra), pp. 186-187; anzi, riprendendo l'ipotesi del Grosso di (a rigore di logica possibile) esperimento separato delle azioni, Burdese suppone che nel caso concreto il marito non avesse fatto valere il suo diritto, e cioè non avesse esercitato la actio empti a sua disposizione, essendo stato poi soluto matrimonio convenuto nella actio rei uxoriae intentata dalla ex-moglie. Ulpiano quindi, in base al criterio del bonum et aequum a cui la actio rei uxoriae si ispira, avrebbe negato l'azione alla moglie, il cui comportamento 
Ammettendosi in teoria, come proposto da Grosso, l'esperimento separato delle azioni, così come, al contrario di $\mathrm{Kaser}^{96}$ e della communis opinio, che la moglie sopportasse il rischio già prima della sua mora, e cioè quando ancora non fosse stata fatta la traditio della cosa, la decisione di Ulpiano potrebbe trovare una nuova spiegazione. Nella actio empti, il marito-compratore, in base alla mora della donna, avrebbe potuto ottenere un risarcimento superiore all'effettivo valore della res: se si suppone, come si è detto, la applicazione nella fattispecie del "Traditionsprinzip", e la conseguente attribuzione del periculum alla moglie già prima della mora, questa avrebbe l'effetto di aumentare il valore del danno da risarcire (il riferimento ad un tale effetto per quanto riguarda, in generale, la funzione della mora in tema di periculum rei venditae, è fatto da Seckel-Levy, ${ }^{97} \mathrm{i}$ quali però lo rifiutano); nella actio rei uxoriae invece la moglie, come è ovvio, chiederebbe soltanto il valore rappresentato dalla aestimatio precedentemente áccordata.

In conseguenza si potrebbe concludere che il valore da ottenere con la actio empti sarebbe stato superiore a quello della aestimatio da reclamare con la actio rei uxoriae. Ed ecco dunque, probabilmente, perchè (--accettandosi la nostra

(convenire il marito per la restituzione della cestimatio non essendo stata prima, a sua volta, convenuta dal marito per il perimento della cosa a lei imputabile considerata la sua mora) non gli era sembrato giusto ed opportuno.

Questa congettura di Burdese ci sembra accettabile. Manca però una importante precisione, riguardante la possibilità (teorica) di valori diversi di risarcimento, come si dirà in seguito. La decisione di Ulpiano comunque, conclude Burdese, si giustificherebbe e si spiegerebbe in base al meccanismo proprio della dos aestimata, e cioè senza necessità del richiamo al regime della compravendita il quale nella fattispecie risulterebbe superfluo.

Anche non accettando i nostri argomenti di ordine sostanziale contro la (pretesa) forza probante di questo testo in favore della classicità del principio periculum est emptoris, questa conclusione di Burdese potrebbe comunque servire -anche se con giustificazione e obiettivi diversi dai nostri- allo scopo di escludere questo passo dalla discussione in tema di periculum rei venditae, e cosi, per conseguenza, anche dell'elenco delle testimonianze testuali richiamate dalla communis opinio a sostegno della tesi dominante [pur tenendo conto del fatto che il Burdese, dal canto suo, sembra aderire alla dottrina dominante in materia di periculum rei venditae - cf. "Aestimatio dotis" cit. (nota 28 sopra), pp. 187-188, n. 74]. D'altra parte, ammessa ancora quella conclusione, sarebbe risparmiata ai fautori della tesi contraria, come Haymann, la fatica di dimostrare l'alterazione sostanziale del passo in esame, ipotesi contro cui appunto si esprime Burdese -"Aestimatio dotis" cit. (nota 28 sopra), p. 187, n. 72

\section{Cf. I'ultima nota sopra}

97. Die Gefahrtragung cit. (nota 8 sopra), p. 258: "Wäre das Traditionsprinzip das römische, so müßte die Gefahr bis zur Übergabe beim Verkaüfer sein, gleichviel ob er sich im Verzug befànde oder nicht, und sein Verzug könnte nur die eine Wirkung haben, die Einbuße, die er durch zufälligen Untergang der Sache erleidet, ex fide bona zu einer Ersatzpflicht gegenüber dem Käufer zu steigen" 
tesi, contraria a quella della communis opinio, secondo cui in questo passo -o in tema di dos aestimata-, la moglie sopportava il periculum quando ancora non fosse stata fatta la traditio--), Ulpiano, nell'ipotesi di mora della moglie, decide "actionem eam habere non puto", mantenendo a carico suo il danno: data la (teoricamente) possibile disparità tra i valori di risarcimento, il giurista vorrebbe sottolineare che, in caso di mora della moglie, valeva la soluzione (diversa da quella della vendita) che limitava per lei il danno da subire unicamente al valore della aestimatio.

Da ultimo, non si deve dimenticare che tale soluzione di Ulpiano, così spiegata, corrisponderebbe esattamente ad una tendenza (riconosciuta, come s'è già detto, ${ }^{98}$ da grande parte della dottrina) del diritto classico in tema di dos aestimata secondo la quale nonostante l'equivalenza aestimatio=venditio, le regole della compravendita non venivano applicate dai giuristi classici in tutti i loro effetti nel senso che la causa dotale esercitava comunque la sua efficacia.

Il passo dunque in conclusione deve essere sottomesso ad una duplex interpretatio: mentre per i compilatori l'attribuzione per argumentum e contrario dei rischi al marito-compratore risultava dell'applicazione del principio generale periculum est emptoris, per Ulpiano invece in origine la decisione, cosi come l'abbiamo appena spiegata, presupponeva l'impiego del principio della traditio, materializzatosi nella regola contraria periculum est venditoris o mariti.

\section{IV.2 L'esegesi di Pomponio 14 ad. Sab., D. 23,3,15}

In questo frammento dunque, come si è già visto, Pomponio, secondo l'interpretazione della dottrina dominante, ${ }^{99}$ introdurrebbe espressamente il risultato soltanto indirettamente dedotto --vale a dire, per argumentum e contrario-- dal passo anteriore di Ulpiano: "si per eam non stetisset" e cioè non verificandosi la mora della moglie nella consegna della res dotalis, il marito=compratore dovrà sopportare il danno già prima della traditio, in base al principio periculum est emptoris.

98. Cf. fls. 5-6 e n. 23 sopra.

99. Cf. da ultimo in questo senso, ad esempio, il Streicher, "Periculum dotis" cit. (nota 28 sopra), p. 10: "Dagegen verbleit es nach Pomponius beim periculum des Mannes, wenn die Frau an der Verzögerung bei der Übergade kein Verschulden trifft" 


\section{IV.2.1 Difetti formali e sostanziali}

Il passo sembra a Haymann ${ }^{100}$ spurio od almeno (ammessa la sua sostanziale genuinità) deviato dal suo contesto originale ad opera dei compilatori : indizi in questo senso sarebbero, "sprachlich" dall'impiego del piùcheperfetto congiuntivo "stetisset" accompagnato dall'indicativo presente "aufert" nella frase seguente, e "sachlich", il fatto che dalla fattispecie non risulta in nessun modo, che la discussione è fatta nell'ambito della dos, e non propriamente dell'emptio venditio.

I sospetti maggiori comunque ricadono sulla chiusa "quia quod evenit emptoris periculo est": ${ }^{101}$ Seckel-Levy, ${ }^{102}$ seguiti da Streicher, ${ }^{103}$ pensavano ad una "nachgefügte Glosse" o glossa aggiunta posteriormente, oppure ad un accorciamento del testo ("Texverküzung"), rilievi che comunque, secondo gli autori, non avrebbero secondo loro importanza in senso dogmatico. Più radicale il parere di Burdese, ${ }^{104}$ che elimina la chiusa "quia" rell. con una critica di contenuto sostanziale-dogmatico: secondo l'autore, che cerca di dimostrare il carattere spurio della assimilazione aestimatio=venditio accolta nella compilazione, la decisione di. Pomponio si giustificarebbe senza necessità del richiamo al regime della compravendita; essendo il valore di stima oggetto dell'obbligo di restituzione, il marito avrebbe assunto, già a partire dalla conclusione del patto, oppure, eventualmente, dal verificarsi del matrimonio, la responsabilità per il perimento fortuito. Il Burdese riconosce però, aderendo in questo punto alla communis opinio, che la soluzione di addossare i rischi al marito sia stata probabilmente ricollegata dai classici al principio periculum est emptoris.

Un'ultima proposta di interpolazione è fatta dal Betti, ${ }^{105}$ che sostituisce $<$ maritl $>$ a [emptoris]: il rimaneggiamento avrebbe per scopo, da un lato, di rendere omaggio alla giustinianea C. 5,13,1,9c, e dall'altro, di riaffermare, pur fuori dalla sedes materiae, la soluzione periculum est emptoris, ormai elevata a principio generale. La questione trattata nella fattispecie, della quale appunto i compilatori non

100. Cf. Textkrit. II cit. (nota 6 sopra), pp. 131-132.

101. Cf. Index Interpolationum.

102. Die Gefahrtragung cit. (nota 8 sopra), p. 260.

103. "Periculum dotis" cit. (nota 28 sopra), p. 10.

104. "Aestimatio dotis" cit. (nota 28 sopra), p. 187 e n. 74.

105. "Periculum" cit. (nota 7 sopra), p. 149 
si resero conto, non concernerebbe il rischio contrattuale derivato dalla compravendita, bensí una più ampia responsabilità per mancata restituzione della dote, derivata (o implicita, in base ad una interpretazione tipica integrativa) dallo stesso patto della aestimatio. L'efficacia del patto già prima della traditio (salvo in caso di mora della moglie), e cioè l'attribuzione del danno al marito già prima della consegna, si spiegerebbe quindi, conclude Betti, non con un artificioso avvicinamento alla vendita, "ma proprio col senso che gli si riconosce come patto di reponsabilità" (vale a dire, bensí con fondamento in questo proprio patto di responsabilità).

Il testo invece a nostro parere è genuino ${ }^{106}$--sia dal punto di vista formale che sostanziale-- e rappresenta una prova indiretta della importanza della traditio in tema di rapporti tra periculum rei venditae e periculum dotis aestimatae, come si cercherà di dimostrare in seguito.

IV.2.2 - Il contesto originale della fattispecie e l'ipotesi di Haymann di "Annahmeverzug" del marito-compratore

Per quanto riguarda il contesto originale in cui il testo di Pomponio si inseriva, é probabile, come pensa Burdese, ${ }^{107}$ che, data la sedes materiae, si trattasse --così come anche sembra accolto nella compilazione (ammessa como verosimile le proposte di ricostruzione per il "in dotem dederit" del testo di Ulpiano)-- di costituzione di dos aestimata mediante mancipatio non seguita della consegna contestuale oppure per mezzo di dotis dictio. Pomponio quindi in origine, come ritiene anche Miquel, ${ }^{108}$ avrebbe dovuto risolvere una fattispecie analoga a quella di Ulpiano. $^{109}$

106. Nello stesso senso sembra il Miquel, "Periculum locatoris" cit. (nota 2 sopra), p. 155. Non è però del tutto da escludere la proposta di Betti, pur non essendo di grande importanza per l'esegesi del passo (cf. nota 105 infra).

107. "Aestimatio dotis" cit. (nota 28 sopra), p. 187.

108. "Periculum locatoris" cit. (nota 2 sopra), p. 155.

109. Senza ragione a nostro parere si esprime contro questa proposta di ricostruzione il Calonge, "Aestimatio dotis" cit. (nota 39 sopra), pp. 19-29, n. 40, secondo cui il passo di Ulpino "nada tiene que ver con D. 23,3,15 (Pomp. 14 ad Sab.), en el que Pomponio se refiere a la compraventa...y que fue aproximado al texto de que nos venimos ocupando por los justinianeos bajo su criterio: aestimatio venditio est" II frammento 15, come giustamente ricostruisce il Lenel, era probabilmente inserito con il titolo De iure dotium del libro 14 dei comentari ad Sab., e sottotitolo De dote aestimata aliisque dotis constituendae modis, nel quale appunto Pomponio faceva il ragionamento parallelo tra 
Accertato il probabile contesto originale del passo, resta infine i] problema di determinare il significato di questa decisione in tema di periculum rei venditae/dotis aestimatae.

Non ci pare che questo testo, come vuole la communis opinio, rappresenti una conferma dell'applicazione del (preteso) principio periculum est emptoris, per cui il marito-compratore sopportava i rischi già prima della traditio. Dice Pomponio: "Si per eam non stetisset,..." e cioè "se non fosse dipeso da lei". Ma che cosa non doveva dipendere dalla moglie? Si presuppone, come in D. 18,6,13, "quo minus traderentur", vale a dire, che non dipendesse da lei la mancata tradizione, e cioè "se la mora nella traditio non fossi dipesa da lei" Ora, se esiste una mora nella consegna, e questa mora, come si dichiara nel testo non è di responsabilità della moglie, non resta altro che pensare, in alternativa, ad una mora del marito.

Ci sembra dunque abbastanza verosimile la proposta avanzata dal Haymann ${ }^{110}$ di una "Annahmeverzug" o mora d'accettazione del marito-compratore, motivo per cui appunto Pomponio probabilmente addossava a quest'ultimo il periculum. Tale supposizione, secondo Haymann, sarebbe rafforzata anche dalla espressione "ac si tradidisset" e cioè "come se (la moglie) avesse effettuato la tradizione" cosa non possibile a causa della mora d'accettazione del marito. L'impiego di quest'espressione, concludeva l'autore, sarebbe interamente senza senso dal punto di vista dei compilatori: ammesso come giustinianeo il principio periculum est emptoris (Inst. 3,23,3) non ci sarebbe affatto bisogno di dichiarare "ac si tradidisset" poichè nel pensiero bizantino, indipendentemente dal verificarsi o non della traditio, il marito-compratore già sopportava il periculum dalla conclusione del negozio. ${ }^{111}$

aestimatio dotis e venditio (la cui assimilazione dunque ci pare genuina, come si è già detto). Cf. n. 88 sopra.

110. Textkrit. II cit. (nota 6 sopra), pp. 131-132. L'accompagnano il Beseler, Romanistische Studien cit. (nota 78 sopra), p. 305 (che propone "Quod si per <maritum steterit, quominus res traderetur> al posto di "Quod si per [eam non sielisset]]), il Konstantinowitch, Le "periculum" cit. (nota 6 sopra), pp. 185 e 265, e l'ayiter, "Aestimatio dotis" e compravendita come concetti di interpretazione tra i giuristi classici, in Annali dell'Università di Ankara 6 (1954-1955), pp. 144 ss. (i due ultimi studi però non mi sono stati accessibili). Contro si esprimono specialmente Seckel-Levy, Die Gefahrtragung cit. (nota 8 sopra), p. 260, n. 3 (seguito dall'appleton, Les risques dans la vente et les fausses interpolations [cont.], in RH 6 (1927), p. 198), che ritengono la proposta haymanniana di mora d'accetazione del marito "ohne jeden Anhalt"

111. Cf. Textkrit. I/ cit. (nota 6 sopra), p. 132: "Wie dem auch sei, jedenfalls ist das von den Kompilatoren stehengelassene ac si tradidisset von ihrem Standpunkt aus sinnlos. Denn wenn der 
L'ipotesi che Pomponio nel contesto originale del passo pensasse ad una mora del marito-compratore sembra essere stata già in antico pressuposta da Accursio. Egli ${ }^{1 / 2}$ infatti, in una nota al testo di Pomponio, rimanda alla "l. illud" del titolo De periculo et commodo reivenditae, e cioè appunto Pomp. 31 ad Quintum Mucium, D. 18,6,18, ${ }^{113}$ fattispecie in cui lo stesso Pomponio si occupa degli effetti risultanti dalla mora, iniziando la trattazione proprio con quella del compratore "Illud sciendum est, cum mora emptor adhibere coepit..."-

La prova infine della tesi della mora del marito-compratore in D. 23,3,15 ci sembra pure risultare dall'esame parallelo del famosissimo passo dei lecti empti distrutti dall'edile, Paul. 3 Alf. epit., D. 18,6,13 (nonchè da D.18,6,15 pr.) già riferiti. ${ }^{1 / 4}$ Tutti i tentativi della communis opinio di conciliare questo passo con il (preteso) principio classico periculum est emptoris sembrano falliti a nostro avviso: anche in questo testo Paolo attribuisce il periculum al compratore quando la cosa sia già stata consegnata a quest'ultimo oppure quando dipenda da lui la mancata tradizione ("per eum stetisset quo minus traderentur"), e cioè appunto quando egli si trovi in mora (soluzione riconfermata dallo stesso giurista nel testo seguente, D. 23,3,15 pr.: "Quod si neque traditi essent neque emptor in mora fuisset quo minus

Käufer schon mit dem Vertragsschluß die Gefahr trägt, so hat es keinen Sinn zu sagen, daß, falls Leistungsverzug der Frau nicht vorliegt, diese auch bei Verlust der Dotalsache die aestimatio fordern könne, wie wenn sie diese bereits tradiert hätte" -sottolinea dallo stesso autore). "Im Sinn der Byzantiner", conclude com ragione il Haymann, Textkrit. II cit. (nota 6 sopra), p. 132, "ist ja die geschehene Tradition ganz belanglos"

Altri passi in tema di periculum rei venditae -come C. 4,48,4 e 6-, in cui c'è anche riferimento alla (assenza di) mora del venditore, sono ugualmente interpretati da Haymann come se, in alternativa, fosse presupposta la mora del compratore. "Non essendo in mora il venditore" -sine mora venditoris significherebbe "essendo in mora il compratore" -propter moram emptoris- (quest'ultima espressione è di Seckel-Levy, Die Gefahrtragung cit. (nota 8 sopra), p. 259, n. 3, i quali, come si è sopra detto, combattono l'interpretazione di Haymann).

Per quanto riguarda questi due rescritti non possiamo esprimere (ancora) una opinione, giacchè non sono oggetto di questo studio. Per quanto concerne invece il testo di Pomponio, la cui decisione tra l'altro è impostata in modo diverso (non c'è riferimento al "sine mora venditoris", bensì al "quod si per eam non stetisset", che ci sembra cosa ben diversa), ci pare giusta l'ipotesi avanzata da Haymann.

112. Glossa ad h.l., ed. Lugduni, 1593.

113. "Illud sciendum est, cum mora emptor adhibere coepit, iam non culpam, sed dolum malum tantum praestandum a venditore. Quod si per venditorem et emptorem mora fuerit. Labeo quidem scribit emptori potius nocere quam venditori moram adhibitam, sed videndum est, ne posterior mora damnosa ei sit. Quid enim si interpellavero venditorem et non dederit id quod emeram, deinde postea offerente illo ego non acceperim? Sane hoc casu nocere mihi deberet. Sed si per emptorem mora fuisset, deinde, cum omnia in integro essent, venditor moram adhibuerit, cum posset se exsolvere, aequum est posteriorem moram venditori nocere"

114. Cf. fls. $8-9$, nn. 23 e 24 sopra. 
traderentur, venditoris periculum erit"). Da notarsi che Paolo, così come Pomponio in D. 23,3,15, adopera addirittura la stessa espressione "si per eum stetisset" per indicare la mora del compratore (anche se nel passo di Pomponio questa espressione si deduca a contrariis).

L'interpretazione dunque di Pomponio D. 23,3,15 deve essere fatta alla luce delle decisioni di Paulo in D. 18,6,13 e D. 18,6,13,15 pr.-1. Anche in questi passi possiamo trovare la soluzione "emptoris periculum esse placet" e "emptoris esse periculo respondit".

E questa soluzione è chiaramente dovuta, nel pensiero del giurista, non alla applicazione di un "principio generale" periculum est emptoris, bensì semplicemente al fatto che i letti venduti "traditi essent emptori aut per eum stetisset quo minus traderentur" (D. 18,6,13), oppure che il legno comprato peri "postquam tradita esset" (D. 18,6,15,1). La ratio decidendi quindi è concentrata sull'elemento rappresentato dalla traditio.

Ci pare dunque che l'esegesi del passo di Pompônio debba essere impostata esattamente nella stessa maniera di quella di Paolo. Anche in D. 23,3,15 quindi la giustificazione presentata da Pomponio alla sua decisione -"quia quod evenit emptoris periculo est"- presuppone una specifica motivazione o causa scaturita dalla specifica relazione fattuale: nel caso, la non avvenuta traditio, dovuta alla "Annhameverzug" o mora d'accettazione dello stesso marito, e non all'applicazione di un "principio generale" d'attribuzione dei rischi ${ }^{15}$ al maritocompratore già prima della consegna (periculum est emptoris o mariti). ${ }^{116}$

115. Vale a dire, i rischi risultati dal perimento o deterioramento fortuito della res dotalis aestimata. In verità, non esiste nelle nostre fonti una soluzione esplicita per l'ultimo caso, quello di semplice deterioramento: tanto i gruppi di passi D. 23,3,10,4-5 quanto D. 23,3,14-15 riguardano unicamente fattispecie concernenti casi di perimento. In tale situazione delle fonti, si può forse dedurre che la soluzione impiegata sia stata la stessa destinata alle ipotesi di perimento. Esatamente dunque, su questo punto, lo Streicher, "Periculum dotis" cit. (nota 28 sopra), p. 10, anche se egli parte da un presupposto diverso dal nostro, e cioè che il marito sopportava il periculum a partire dalla realizzazione delle nozze e non, come riteniamo, a partire dalla traditio.

116. In questa ricostruzione del passo, non ci sembra da escludere del tutto la proposta di Betti, "Periculum" cit. (nota 7 sopra), p. 149, già menzionata, che suggerisce "mariti" al posto di "emptoris" L'accertamento di quest'eventuale interpolazione però non ha comunque importanza di ordine sostanziale, giacchè dogmaticamente la soluzione non cambia (cf. nello stesso senso il Streicher, "Periculum dotis" cit., nota 28 sopra, p. 10). 


\section{$\mathrm{V}$ Conclusione}

La questione del periculum rei venditae dovrebbe dunque a nostro avviso essere impostata in un modo diverso da quello usuale negli ultimi anni.

La soluzione periculum est emptoris ha assunto il valore di un "principio generale" soltanto con Giustiniano (come appunto dichiarato in Inst. $3,23,3)$. Per il diritto classico invece è probabile, come di recente già proposto da Talamanca, ${ }^{117}$ che le soluzioni in materia di rischio contrattuale nella compravendita fossero differenziate, a seconda dei diversi contesti problematici e delle diverse personalità dei giuristi. Il diritto classico dunque a nostro avviso sarebbe stato contrasegnato, in tema di periculum rei venditae, da un "elastische Gebilde", in cui, d'accordo con i differenti contesti delle fattispecie, si attribuiva il danno per il perimento fortuito o deterioramento della cosa venduta ora al compratore (periculum est emptoris), ora al venditore (periculum est venditoris).

Tra questi diversi contesti problematici, ci pare che quello contrassegnato dal principio della traditio --e cioè la cosiddetta soluzione germanica (adottata pure dal nostro Codice Civile brasiliano), in cui il compratore sopporta i rischi solo a partire dal momento della consegna-- abbia svolto un fondamentale ruolo in non pochi passi.

Fra questi passi in cui ci è apparso determinante, ai fini della decisione, il principio della tradizione, sono da elencare appunto quelli principali in tema di periculum dotis aestimatae, (richiamati comunemente dalla dottrina, data I'assimilazione aestimatio=venditio presente nelle nostre fonti): Ulp. 34 ad Sab., D. $23,3,10,4-5$, in cui non si tratta lo specifico problema dell'attribuzione del periculum tra il momento della realizzazione della condizione (le nozze) e l'effettiva consegna materiale della res aestimata; e --principalmente- Ulp. 34 [ad ed.]<ad Sab.>, D. 23,3,14, e Pomp. 14 ad Sab., D. 23,3,15, in cui sembra probabile addossare il periculum al marito-compratore soltanto a partire dalla traditio fatta dalla moglievenditore, oppure dalla "Annahmeverzug" o mora d'accettazione del primo. 